\title{
Algorithm Summary and Evaluation: Automatic Implementation of Ring- down Analysis for Electromechanical Mode Identification from Phasor Measurements
}
N Zhou
$S$ Jin
Z Huang
$\mathrm{J}$ Lin
F Tuffner
M Hauer

February 2010

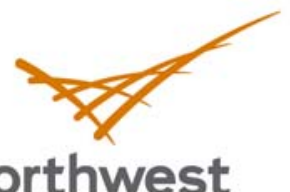

Pacific Northwest

NATIONAL LABORATORY

Proudly Operated by Battelle Since 1965 


\title{
DISCLAIMER
}

This report was prepared as an account of work sponsored by an agency of the United States Government. Neither the United States Government nor any agency thereof, nor Battelle Memorial Institute, nor any of their employees, makes any warranty, express or implied, or assumes any legal liability or responsibility for the accuracy, completeness, or usefulness of any information, apparatus, product, or process disclosed, or represents that its use would not infringe privately owned rights. Reference herein to any specific commercial product, process, or service by trade name, trademark, manufacturer, or otherwise does not necessarily constitute or imply its endorsement, recommendation, or favoring by the United States Government or any agency thereof, or Battelle Memorial Institute. The views and opinions of authors expressed herein do not necessarily state or reflect those of the United States Government or any agency thereof.

\author{
PACIFIC NORTHWEST NATIONAL LABORATORY \\ operated by \\ BATTELLE \\ for the \\ UNITED STATES DEPARTMENT OF ENERGY \\ under Contract DE-AC05-76RL01830
}

Printed in the United States of America
Available to DOE and DOE contractors from the Office of Scientific and Technical Information, P.O. Box 62, Oak Ridge, TN 37831-0062; ph: (865) 576-8401 fax: (865) 576-5728
email: reports@adonis.osti.gov

Available to the public from the National Technical Information Service, U.S. Department of Commerce, 5285 Port Royal Rd., Springfield, VA 22161 ph: (800) 553-6847 fax: (703) 605-6900 email: orders@ntis.fedworld.gov online ordering: http://www.ntis.gov/ordering.htm 


\section{Algorithm Summary and Evaluation: Automatic Implementation of Ringdown Analysis for Electromechanical Mode Identification from Phasor Measurements}

$\begin{array}{ll}\text { N Zhou } & \text { S Jin } \\ \text { Z Huang } & \text { J Lin } \\ \text { F Tuffner } & \text { M Hauer }\end{array}$

February 2010

Prepared for

Public Interest Energy Research (PIER), California Energy Commission via U.S. Department of Energy

Contract DE-AC05-76RL01830

Pacific Northwest National Laboratory

Richland, Washington 99352 



\section{Preface}

The California Energy Commission's Public Interest Energy Research (PIER) Program supports public interest energy research and development that will help improve the quality of life in California by bringing environmentally safe, affordable, and reliable energy services and products to the marketplace.

The PIER Program conducts public interest research, development, and demonstration (RD\&D) projects to benefit California.

The PIER Program strives to conduct the most promising public interest energy research by partnering with RD\&D entities, including individuals, businesses, utilities, and public or private research institutions.

- $\quad$ PIER funding efforts are focused on the following RD\&D program areas:

- Buildings End-Use Energy Efficiency

- Energy Innovations Small Grants

- Energy-Related Environmental Research

- Energy Systems Integration

- Environmentally Preferred Advanced Generation

- Industrial/Agricultural/Water End-Use Energy Efficiency

- Renewable Energy Technologies

- Transportation

Algorithm Summary and Evaluation: Automatic Implementation of Ringdown Analysis for Electromechanical Mode Identification from Phasor Measurements is an interim report for the Oscillation Detection and Analysis (contract number: 500-07-037, work authorization number: TRP-08-07) conducted by Pacific Northwest National Laboratory. The information from this project contributes to PIER’s Energy Systems Integration Program.

For more information about the PIER Program, please visit the Energy Commission's website at www.energy.ca.gov/research/ or contact the Energy Commission at 916-654-4878. 


\section{EXECUTIVE SUMMARY}

Small signal stability problems are one of the major threats to grid stability and reliability. An unstable oscillatory mode can cause large-amplitude oscillations and may result in system breakup and large-scale blackouts. There have been several incidents of system-wide oscillations worldwide [1]. Of them, the most notable is the August 10, 1996 western system breakup produced as a result of undamped system wide oscillations [2]. To avoid system breakups caused by oscillations, the dynamic stability margin has to be established, which puts limits on the power transfer capacities. Accurate and timely information about the oscillations can help optimize these margin settings so that a grid can be operated at its full capacity while staying within the stability boundary.

There are two basic approaches for estimating power system modes: component-based methods and measurement-based methods. The efforts of building a component-based model are not trivial and thus it is hard to provide real time updates on the oscillation information. In contrast, a measurement-based approach usually requires significantly less effort. The measurementbased method can update the mode estimation based on incoming measurement data. Thus, the measurement-based methods can serve as a good complement to model-based methods in monitoring power system modes in real time.

To achieve desired performance and reduce false/missing alarms with measurement based methods, measurement data should be classified into different categories to be sure that a proper mode identification algorithm can be selected. Applying a mode analysis algorithm on a data set that is not suitable for that particular algorithm may result into degraded performance, and even false or missing alarms. Thus, it is proposed in this report that data be categorized so that a proper mode analysis method can be selected and applied to get timely and accurate mode estimation.

In this report, a recursive Prony algorithm is described for implementing ringdown data detection and analysis. Simulation data are used to show the statistical performance of the algorithm. Field measurement data from Wide Area Measurement System (WAMS) are used to verify the applicability of the proposed algorithm. It is shown that the proposed algorithm can effectively detect the ringdown data. Based on the detection, Prony analysis can be applied to estimate oscillation mode timely and accurately. 
The preparation of this report was conducted with support from the California Energy Commission's Public Interest Energy Research Program through the California Institute of Energy and Environment, and support from the Transmission Reliability Program of the Department of Energy's Office of Electricity Delivery and Energy Reliability.

Technical discussions with the Technical Advisory Committee have been instrumental in the progression of this report. Furthermore, the authors would also like to thank Mr. Jim Cole and Mr. Larry Miller with the California Institute for Energy and Environment, Dr. John Hauer with the Pacific Northwest National Laboratory, and James (Jim) Burns with the Bonneville Power Administration for their help and assistance with this project.

Project support provided by Sue Arey and Kim Chamberlin, both with Pacific Northwest National Laboratory, is gratefully acknowledged.

The Technical Advisory Committee for the project entitled "Oscillation Detection and Analysis", funded by the California Energy Commission's Public Interest Energy Research Program through the California Institute of Energy and Environment, consists of the following academic and industry experts:

- Jeff Dagle, Pacific Northwest National Laboratory

- Soumen Ghosh, California Independent System Operator

- Dmitry Kosterev, Bonneville Power Administration

- Bill Mittelstadt, Retiree, Bonneville Power Administration

- $\quad$ Phil Overholt, Department of Energy

- Manu Parashar, Electric Power Group

- John Pierre, University of Wyoming

- Dan Trudnowski, Montana Tech of the University of Montana

- Matthew Varghese, California Independent System Operator. 
Table of Contents

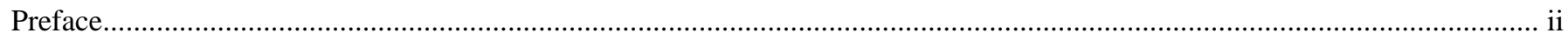

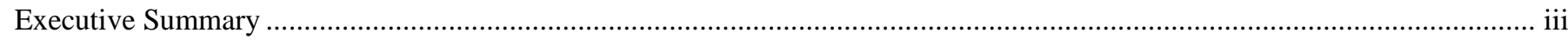

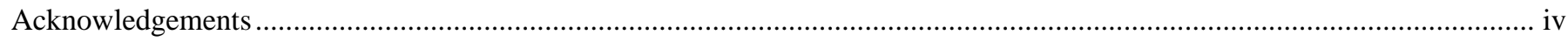

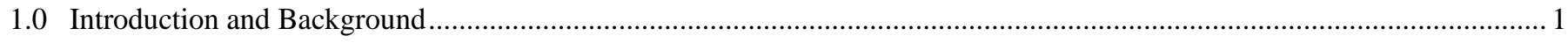

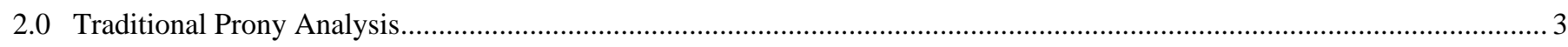

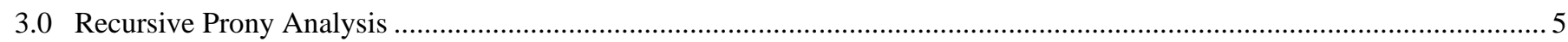

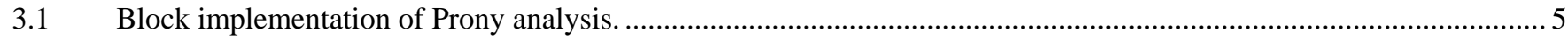

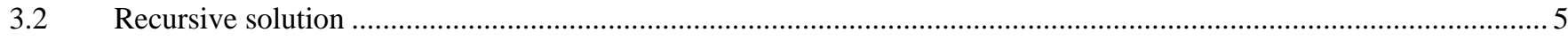

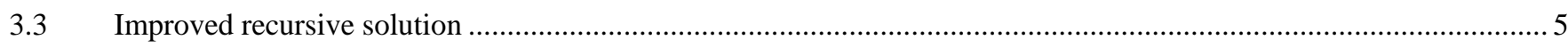

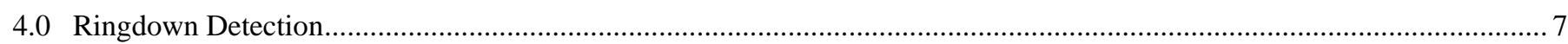

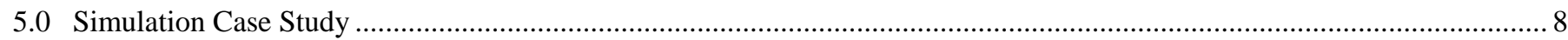

6.0 Field Measurement Data Case Studies ......................................................................................................................... 13

6.1. Oscillations detected right before WECC Break up on Aug 10th, 1996................................................................ 13

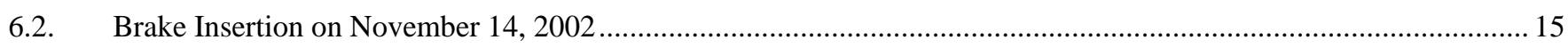

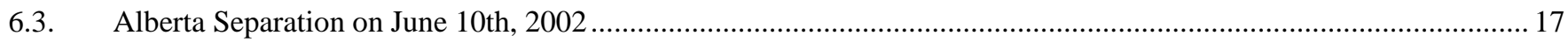

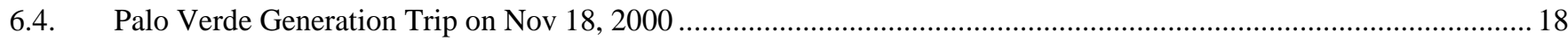

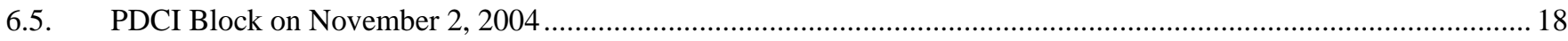

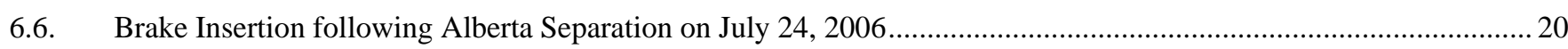

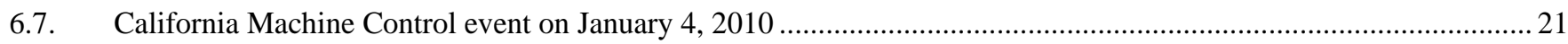

7.0 Conclusions and Future Work ................................................................................................................................ 24

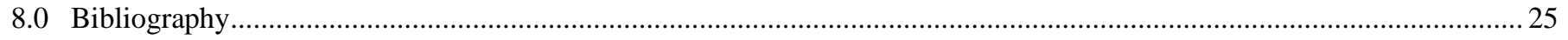




\section{Table of Figures}

Figure 1-1 - Flow chart for integration of the proposed oscillation detection algorithm. ........................................................ 2

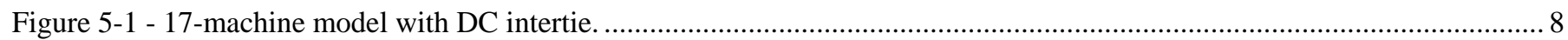

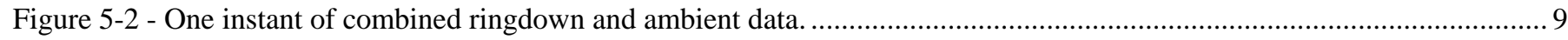

Figure 5-3 - Relative noise levels for 100 data sets of Monte Carlo simulation. ........................................................................ 9

Figure 5-4 - Measurement energy for 100 data sets of Monte Carlo simulation........................................................................ 10

Figure 5-5 - Prediction correction for 100 data sets of Monte -Carlo simulation.................................................................. 10

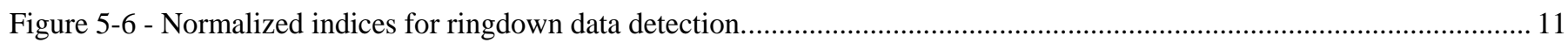

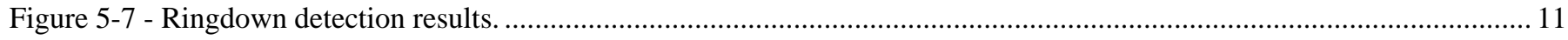

Figure 5-8 - Mode estimation results from detected ringdown data.................................................................................... 12

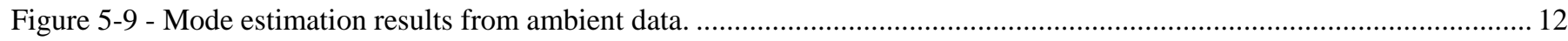

Figure 6-1 - Recorded real power flow from Malin to Round Mountain with detected oscillation. (Reference time: August 10,

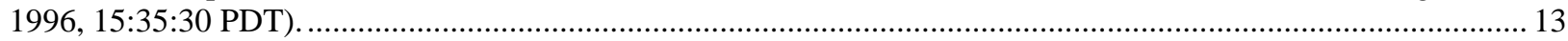

Figure 6-2 - Detail plot of the first oscillation detected after the Keeler-Allston line trips........................................................ 14

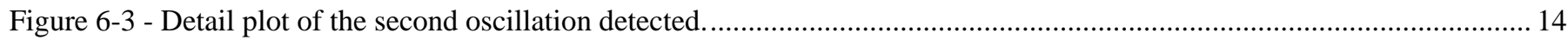

Figure 6-4 - Detail plot of the third and fourth oscillations detected after the Ross-Lexington line trips................................... 15

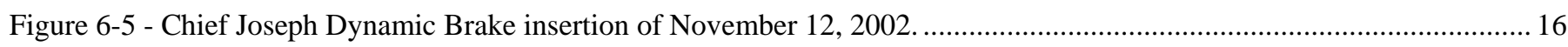

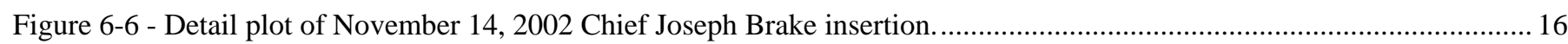

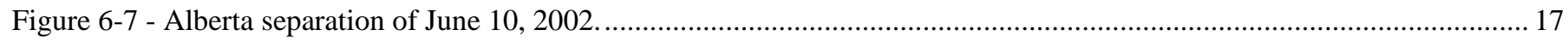

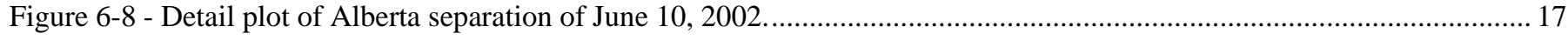

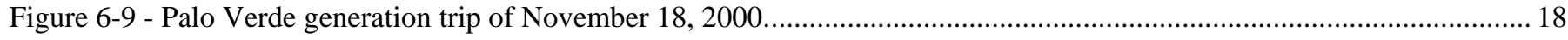

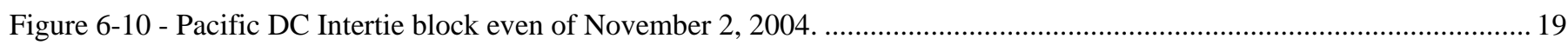

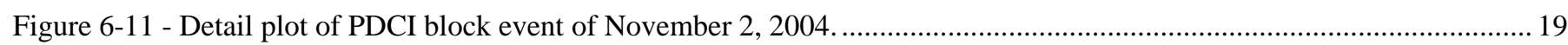

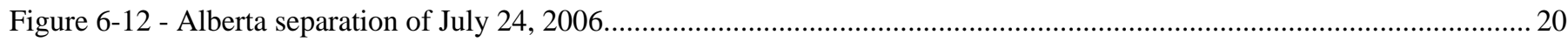

Figure 6-13 - Detail plot of line fault and brake insertion shortly after Alberta separation of July 24, 2006...............................20

Figure 6-14 - Detail plot of Colstrip generation loss and secondary event after Alberta separation of July 24, 2006..................21

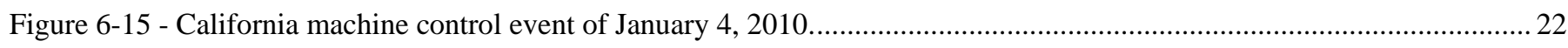

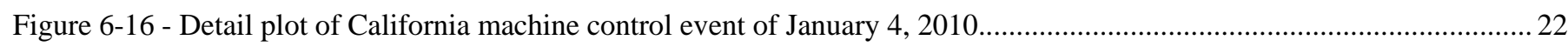

Figure 6-17 - Detail plot of California machine control event of January 4, 2010 - 24 hour data.................................................. 23

\section{Table of Tables}

Table 5-1 - Inter-area modes of 17-machine system 


\subsection{INTRODUCTION AND BACKGROUND}

Small signal stability problems are one of the major threats to grid stability and reliability. An unstable oscillatory mode can cause large-amplitude oscillations and may result in system breakup and large-scale blackouts. There have been several incidents of system-wide oscillations worldwide [1]. Of them, the most notable is the August 10, 1996 western system breakup produced as a result of undamped system wide oscillations [2]. To avoid system breakups caused by oscillations, the dynamic stability margin has to be established, which puts limits on the power transfer capacities. Accurate and timely information about the oscillations can help optimize these margin settings so that a grid can be operated at its full capacity while staying within the stability boundary.

To provide timely information about grid oscillation, extensive studies have been carried out to identify power system modes. Modes are the eigenvalues of linearized power system models. Generally, there are two basic approaches for estimating power system modes: component-based methods and measurement-based methods. With the component-based method, the nonlinear differential equations governing the system are linearized around an operating point. The power system modes are then obtained through eigenvalue analysis [3]. On the other hand, for a measurement-based method, a linear model is estimated from direct system measurements [4].

An important aspect to remember is that for a large complex power system, the efforts of building a component-based model are not trivial. For example, an initial effort was made by [2] to build a component-based model for simulating the Western Electricity Coordinating Council (WECC) reaction right before the breakup of August 10, 1996. The simulation data from the initial model did not match the field measurement data. Matched simulation and measurement results were only achieved after extensive studies. In contrast, a measurement-based approach usually requires significantly less effort. The measurement-based method can update the mode estimation based on incoming measurement data. Thus, the measurement-based methods can serve as a good complement to model-based methods in monitoring power system modes in real time.

There exist several measurement-based small signal stability analysis algorithms, which have been developed and studied [4]-[15]. Performance studies of the existing small signal stability analysis algorithms have been carried out. There are no comprehensive comparisons of all the algorithms. One reason for the lack of this comparison is likely tied to algorithm performance and its likelihood to be situation-dependent. One algorithm would perform better under some circumstances, while others may perform better in other circumstances. Ultimately, it is conjectured that the right combination of algorithms needs to be used to support real-time power grid operation [5]. Applying a mode analysis algorithm on a data set that is not suitable for that particular algorithm may result into degraded performance, and even false or missing alarms.

To achieve desired performance and reduce false/missing alarms, measurement data should be classified into different categories to be sure that a proper mode identification algorithm can be selected. In general, field measurement data can be classified into two categories: typical and non-typical data. Typical data is the data that carries system mode information and can be described by the model structure used by an identification algorithm. In contrast, non-typical data does not carry information about system modes and cannot be described by a general linear model.

Commonly encountered non-typical data points include, but are not limited to, missing data and outliers. Missing data are often dropped data points, which may result from temporary communication and measurement device failure. Outliers are values that significantly deviate from normal values. Outliers may result from a serious disturbance and/or sensor failure. In general, data that cannot be described by the adopted model structure is considered non-typical data. For example, transient data right before ringdown signals is also considered non-typical, namely because it cannot be described by a linear prediction model.

Commonly encountered typical data can include, but are not limited to, ambient data, ringdown oscillations, and probing data. Ambient data is obtained when a system is working under an equilibrium condition, and the major disturbance is from smallamplitude random load changes [7]. Ringdown oscillation data occurs after some major disturbance, such as a line tripping, and results in observable oscillations [4]. Probing data is obtained when low-level pseudo-random noise is intentionally injected into the system to test the system performance [12].

Note that these three types of data carry different levels of mode information density. As shown in [14], the ringdown oscillation data carries the highest level of information density. The mode estimation converges fast to the true values. As such, it is valuable to identify oscillation data from other signals. An identified ringdown oscillation can help: select right algorithm, reduce the mode estimation time, and provide an indication of the disturbance events.

Figure 1-1 shows a flow chart for integrating the ringdown oscillation analysis into a mode analysis study. After PMU data is obtained, the first step is to classify the data into typical and non-typical data according to a prediction error model [13]. Then, the typical data is checked for presence of a ringdown oscillation. If a ringdown oscillation is detected, then the proposed recursive Prony analysis can be applied for mode identification. If the features of the data fits assumptions of another algorithm (e.g. ambient assumption), the corresponding algorithm will be applied. The mode information is displayed after an identified model passes model validation [16]. 


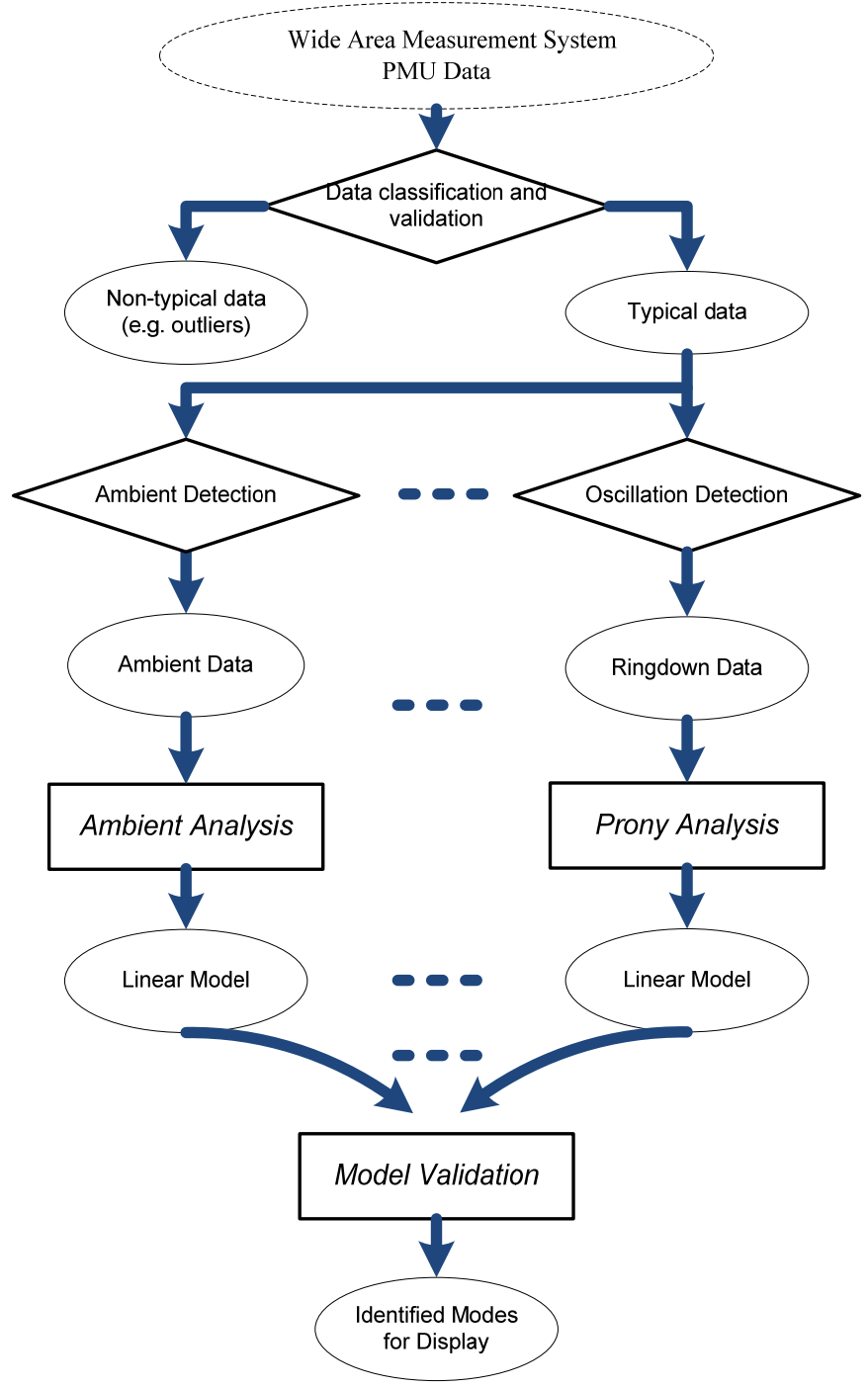

Figure 1-1 - Flow chart for integration of the proposed oscillation detection algorithm.

The purpose of this report is to develop a recursive Prony analysis algorithm for on-line estimation of modes, to propose a ringdown detection method, and to apply the proposed method to simulation and field measurement data. The report is organized in the following way. In Section 2.0, a traditional Prony analysis is reviewed. In Section 3.0, a recursive Prony analysis algorithm is developed. In Section 4.0, a ringdown detection method is proposed. In Section 5.0, simulation data sets from a 17machine model are used to evaluate the performance of the proposed algorithm and method using the Monte-Carlo method. Section 6.0 presents a variety of field measurement cases and the proposed algorithm's results. Finally in Section 7.0, some conclusions and future work are discussed. 


\subsection{Traditional Prony ANALysis}

As discussed in [4], Prony analysis can be used to estimate the system modes from ringdown data. If a system can be described by a linear state space model, the homogeneous responses of the system are a sum of exponentially damped sinusoidal signals, which is also known as ringdown data. The ringdown data can be described as

$$
y[j]=\sum_{i=1}^{n_{\lambda}} c_{i} z_{i}^{j}
$$

$$
\text { for } j=k, k+1, k+2, \ldots, k+N-1 \text {. }
$$

Here, $z_{i}=\exp \left(\lambda_{i} \Delta t\right)$ and $y[j]$ is the ringdown data at time $j \Delta t$. The symbol $\Delta t$ stands for the sampling interval. The symbol $\lambda_{i}$ represents the $i^{\text {th }}$ eigenvalue, $c_{i}$ is the amplitude of $i^{\text {th }}$ mode, and $n_{\lambda}$ is the total number of eigenvalues. Symbol $k$ in (1) stands for the starting time of ringdown, and $N$ represents the total number of ringdown data points.

Denote $\hat{y}[j]$ as the measurement from ringdown data described by (1). Note that $\hat{y}[j]$ contains measurement and process noise in addition to the ringdown signal. According to Prony analysis proposed by [4], power system modes can be identified through the following procedure.

First, calculate $\theta[k]$ by solving the following equations in the least square sense.

$$
\vec{y}[k]=H[k] \theta[k]+\vec{e}[k]
$$

where

$$
\begin{aligned}
& \vec{y}[k]=\left[\begin{array}{c}
\hat{y}[k+n] \\
\hat{y}[k+n+1] \\
\vdots \\
\hat{y}[k+N-1]
\end{array}\right] \\
& H[k]=\left[\begin{array}{c}
\varphi^{T}[k+n-1] \\
\varphi^{T}[k+n] \\
\vdots \\
\varphi^{T}[k+N-2]
\end{array}\right] \\
& =\left[\begin{array}{cccc}
\hat{y}[k+n-1] & \hat{y}[k+n-2] & \cdots & \hat{y}[k] \\
\hat{y}[k+n] & \hat{y}[k+n-1] & \cdots & \hat{y}[k+1] \\
\vdots & \vdots & \ddots & \vdots \\
\hat{y}[k+N-2] & \hat{y}[k+N-3] & \cdots & \hat{y}[k+N-n-1]
\end{array}\right] \\
& \varphi[j]=\left[\begin{array}{c}
\hat{y}[j] \\
\hat{y}[j-1] \\
\vdots \\
\hat{y}[j-n+1]
\end{array}\right] \\
& \vec{e}[k]=\left[\begin{array}{c}
e[k+n] \\
e[k+n+1] \\
\vdots \\
e[k+N-1]
\end{array}\right] \\
& \theta[k]=\left[\begin{array}{c}
\hat{a}_{1} \\
\hat{a}_{2} \\
\vdots \\
\hat{a}_{n}
\end{array}\right]
\end{aligned}
$$

where $n$ is the number of modes to be estimated. The least-square solution can be described as

$$
\begin{aligned}
& \theta[k]=\min (\vec{y}[k]-H[k] \theta[k])^{T} \Lambda(\vec{y}[k]-H[k] \theta[k]) \\
& \text { for } k \geq 0
\end{aligned}
$$

where

$$
\Lambda=\left[\begin{array}{llll}
\lambda^{N-n-1} & & & \\
& \lambda^{N-n-2} & & \\
& & \ddots & \\
& & & \lambda^{0}
\end{array}\right]
$$


where $\lambda$ is the forgetting factor, which is a positive number slightly smaller than or equal to 1 . In this report, it is chosen as 1 to equally weigh data.

To filter out noise, the number of equations is usually selected to be greater than $2 n$ [4]. In this report, it is chosen as $3 n$. The estimates of $z_{i}$, denoted as $\hat{z}_{i}$, can be estimated as the roots of the polynomial of

$$
\hat{z}^{n}-\left[\hat{a}_{1} \hat{z}^{n-1}+\hat{a}_{2} \hat{z}^{n-2}+\cdots+\hat{a}_{n} \hat{z}^{0}\right]=0
$$

According to [7], the eigenvalues, or the modes, of the system can be estimated as

$$
\hat{s}_{i}=\frac{1}{\Delta t} \ln \left(\hat{z}_{i}\right) \quad \text { for } i=1,2, \cdots, n
$$

Once the eigenvalue $s_{i}$ and $z_{i}$ are identified, the time domain ringdown signal can be reconstructed. The following equation can be solved in least square sense to estimate oscillation amplitude $c_{i}$ denoted as $\hat{c}_{i}$.

$$
\left[\begin{array}{cccc}
\hat{z}_{1}^{0} & \hat{z}_{2}^{0} & \cdots & \hat{z}_{n}^{0} \\
\hat{z}_{1}^{1} & \hat{z}_{2}^{1} & \cdots & \hat{z}_{n}^{1} \\
\vdots & \vdots & \ddots & \vdots \\
\hat{z}_{1}^{N-1} & \hat{z}_{2}^{N-11} & \cdots & \hat{z}_{n}^{N-n-1}
\end{array}\right] \cdot\left[\begin{array}{c}
\hat{c}_{1} \\
\hat{c}_{2} \\
\vdots \\
\hat{c}_{n}
\end{array}\right] \approx\left[\begin{array}{c}
\hat{y}[k] \\
\hat{y}[k+1] \\
\vdots \\
\hat{y}[k+N-1]
\end{array}\right]
$$

The pure ringdown can be constructed as

$$
\left[\begin{array}{c}
\tilde{y}[k] \\
\tilde{y}[k+1] \\
\vdots \\
\tilde{y}[k+N-1]
\end{array}\right] \Delta=\left[\begin{array}{cccc}
\hat{z}_{1}^{0} & \hat{z}_{2}^{0} & \cdots & \hat{z}_{n}^{0} \\
\hat{z}_{1}^{1} & \hat{z}_{2}^{1} & \cdots & \hat{z}_{n}^{1} \\
\vdots & \vdots & \ddots & \vdots \\
\hat{z}_{1}^{N-1} & \hat{z}_{2}^{N-11} & \cdots & \hat{z}_{n}^{N-n-1}
\end{array}\right] \cdot\left[\begin{array}{c}
\hat{c}_{1} \\
\hat{c}_{2} \\
\vdots \\
\hat{c}_{n}
\end{array}\right]
$$

Note that the reconstructed ringdown signal $\tilde{y}[k]$ usually does not perfectly match the measurement $\hat{y}[k]$. The posterior estimation noise can be defined as

$$
\begin{gathered}
\hat{e}[j]=\hat{y}[j]-\tilde{y}[j] \\
\text { for } j=k, k+1, k+2, \ldots, k+N-1 .
\end{gathered}
$$




\subsection{Recursive Prony ANalysis}

A recursive algorithm can help on-line implementation by improving calculation efficiency and reduce memory requirement [16]. In addition, as pointed out by [14], a robust algorithm can be easily designed for a recursive least square method. A recursive Prony analysis algorithm is developed in this section.

\subsection{Block implementation of Prony analysis.}

The traditional block form of implementing Prony analysis is straight forward. By setting the first order derivative of the objective function of equation (8), the block solution can be readily found as

$$
\hat{\theta}[k]=\Phi^{-1}[k] \cdot s[k]
$$

where

$$
\begin{gathered}
\Phi[k] \stackrel{\Delta}{=} H^{T}[k] \Lambda H[k] \\
s[k] \stackrel{\Delta}{=} H^{T}[k] \Lambda \vec{y}[k]
\end{gathered}
$$

Note that

$$
\begin{aligned}
\Phi[k]= & H^{T}[k] \Lambda H[k] \\
= & \lambda \Phi[k-1]+\varphi[k+N-2] \cdot \varphi^{T}[k+N-2] \\
& -\lambda^{N-n} \varphi[k+n-2] \cdot \varphi^{T}[k+n-2] \\
s[k]= & H^{T}[k] \Lambda \vec{y}[k] \\
= & \lambda S[k-1]+\varphi[k+N-2] \cdot y[k+N-1] \\
& -\lambda^{N-n} \varphi[k+n-2] y[k+n-1]
\end{aligned}
$$

where $y[k]$ is the measured data at sampling instance $k$.

\subsection{Recursive solution}

For on-line implementation of Prony analysis, a recursive form is useful. A recursive implementation of Prony analysis can be derived as

where

$$
\begin{aligned}
\hat{\theta}[k] & =\Phi^{-1}[k] \cdot s[k] \\
& =\hat{\theta}[k-1]+\Phi^{-1}[k] \cdot \varphi[k+N-2] \cdot\{y[k+N-1] \\
& \left.-\varphi^{T}[k+N-2] \cdot \hat{\theta}[k-1]\right\}-\lambda^{N-n} \Phi^{-1}[k] \cdot \\
& \varphi[k+n-2] \cdot\left\{y[k+n-1]-\varphi^{T}[k+n-2] \cdot \hat{\theta}[k-1]\right\} \\
& =\hat{\theta}[k-1]+\Phi^{-1}[k] \cdot \varphi[k+N-2] \cdot \varepsilon[k+N-1] \\
& -\lambda^{N-n} \Phi^{-1}[k] \cdot \varphi[k+n-2] \cdot\{y[k+n-1] \\
& \left.-\varphi^{T}[k+n-2] \cdot \hat{\theta}[k-1]\right\}
\end{aligned}
$$

$$
\varepsilon[k+N-1]=y[k+N-1]-\varphi^{T}[k+N-2] \cdot \hat{\theta}[k-1]
$$

is the priori prediction noise. This priori prediction noise is the difference between the measurement at $k+N-1$, and the prediction based on the past ringdown model. Note that the past ringdown model is built based on the measurement taken before $k+N-1$ (not including $k+N-1$ ). The prediction noise serves as an indication of how well the current ringdown model describes the next available data.

To summarize, a recursive Prony analysis algorithm is formed by equations (18), (19) and (20). Updated Prony analysis estimates can be calculated by updating previous estimation using current measurements. The storage requirements and calculation time are all fixed.

\subsection{Improved recursive solution}

Note that (18), (19) and (20) require the calculation of inverse matrix $\Phi^{-1}[k]$, which is a time-consuming computation. The calculation efficiency can be further increased by applying matrix inversion lemma [16] as

$$
[A+B C D]^{-1}=A^{-1}-A^{-1} B\left[D A^{-1} B+C^{-1}\right]^{-1} D A^{-1}
$$

To facilitate notation, define

$$
P[k] \stackrel{\Delta}{=} \Phi^{-1}[k]
$$


After applying the matrix inversion lemma,

$$
\begin{aligned}
& P[k]=\Phi^{-1}[k]=\left\{\begin{array}{l}
\lambda \Phi[k-1]+\varphi[k+N-2] \cdot \\
\varphi^{T}[k+N-2]-\lambda^{N-n} \varphi[k+n-2] \\
\cdot \varphi^{T}[k+n-2]
\end{array}\right\}^{-1} \\
& =Q[k]-\frac{Q[k] \varphi[k+N-2] \cdot \varphi^{T}[k+N-2] Q[k]}{1+\varphi^{T}[k+N-2] Q[k] \varphi[k+N-2]}
\end{aligned}
$$

is obtained, where

$$
Q[k] \stackrel{\Delta}{=}\left(\lambda \Phi[k-1]-\lambda^{N-n} \varphi[k+n-2] \cdot \varphi^{T}[k+n-2]\right)^{-1}
$$

A further application of the matrix inversion lemma to (25) results in

$$
\begin{aligned}
& Q[k] \stackrel{\Delta}{=}\left(\lambda \Phi[k-1]-\lambda^{N-n} \varphi[k+n-2] \cdot \varphi^{T}[k+n-2]\right)^{-1} \\
& =\frac{1}{\lambda}\left\{P[k-1]-\frac{P[k-1] \varphi[k+n-2] \varphi^{T}[k+n-2] P[k-1]}{-\lambda^{-N+n-1}+\varphi^{T}[k+n-2] P[k-1] \varphi[k+n-2]}\right\}
\end{aligned}
$$

Utilizing the derived equations, the recursive Prony analysis at time step $k$ can be implemented by

1) applying equation (26) to calculate $Q[k]$;

2) applying equation (24) to calculate $P[k]$ i.e., $\Phi^{-1}[k]$;

3) applying equation (20) to calculate $\hat{\theta}[k]$. 


\subsection{RINGDOWN DETECTION}

The applicability of the mode identification algorithms (including Prony analysis) rely heavily on the proper use of algorithms. Identification algorithms can provide dependable mode information only when they are applied properly on the right signal types. Prony analysis is known to be applicable to ringdown data. To properly apply Prony analysis automatically, ringdown data should be detected. To reliably identify the ringdown data, three indices are introduced in this section: relative noise level, measurement energy, and prediction correction.

The relative noise level is the percentage of noise with respect to total measurement energy. It is defined as

$$
\text { Relative Noise[k] }=\frac{\sqrt{\left(\sum_{j=k}^{k+N-1} \mid \hat{e}[j]^{2}\right)}}{\sqrt{\left(\sum_{j=k}^{k+N-1} \mid \hat{y}[j]^{2}\right)}}
$$

where $\hat{e}[j]$ is the estimation noise from (14) and $\hat{y}[j]$ is the measurement signal of (3).

As defined in equation (14), the posteriori noise is the measurement component that cannot be explained by the identified ringdown model. Thus, a lower relative noise level indicates a good fit between model and data, and the ringdown assumption may hold. On the other hand, a higher relative noise level indicates that the fit is not so good, and the ringdown assumption may not hold.

For the power system application, the ringdown data is usually caused by a sudden disturbance, such as a brake insertion, line trips, or generator trips. Ringdown data normally carries more energy than ambient data. During such a disturbance interval, the measurement energy, given by

$$
\text { Measurement Energy[k] }=\sqrt{\left(\sum_{j=k}^{k+N-1} \mid \hat{y}\left[j \|^{2}\right)\right.}
$$

increases. A growing signal energy may indicate the arrival of a ringdown disturbance.

Another useful metric for detecting proper algorithm application is the prediction correction, which is defined as

$$
\text { PredictionCorrection }[\mathrm{k}]=\|\varepsilon[k+N-1]-\hat{e}[k+N-1]\|
$$

The prediction correction is the difference between priori prediction noise and posteriori prediction noise. This describes how much adjustment has to be made after a new data point is included. A smaller prediction correction term indicates consistency between current model and the updated model. A large prediction correction indicates significant changes on model after a new measurement data point is included. Inter-area electromechanical modes of a large power system involve many generators. Thus, it is reasonable to assume that the modes do not change significantly during a ringdown procedure. Thus, a smaller prediction correction term is expected for the ringdown data.

For reliably detect ringdown data, the following method is proposed.

1) Arm the ringdown detector, when:

- the relative noise level is lower than a preselected threshold (the threshold is selected to be mean-3*std for the ambient data in this report);

- the measurement energy level exceeds a preselected threshold (the threshold is selected to be mean+10*std for the ambient data in this report);

- and the prediction correction is lower than a preselected threshold (the threshold is selected to be mean+10*std for the ambient data in this report).

2) If the ringdown detector is armed, set the start of ringdown data after the relative noise level reaches a local minimum point.

3) If the start of ringdown data has been set, set end of the ringdown data when the relative noise level exceeds a preselected threshold (the threshold is selected to be mean-3*std for the ambient data in this report).

Note that the recursive Prony algorithm is applied continuously on all the measurement data. However, only when the ringdown data is detected utilizing the above criteria are modes estimated from Prony analysis trusted. 


\subsection{SimULATION CASE STUdy}

Simulation studies are used to evaluate the performance of the proposed recursive Prony analysis algorithm and ringdown data detection method. A 17-machine model (shown in Figure 5-1) is used to generate simulation data for testing the performance of the proposed method. This model has been used in many studies to evaluate performance of mode identification algorithms. A detailed description of the model can be found in [17].

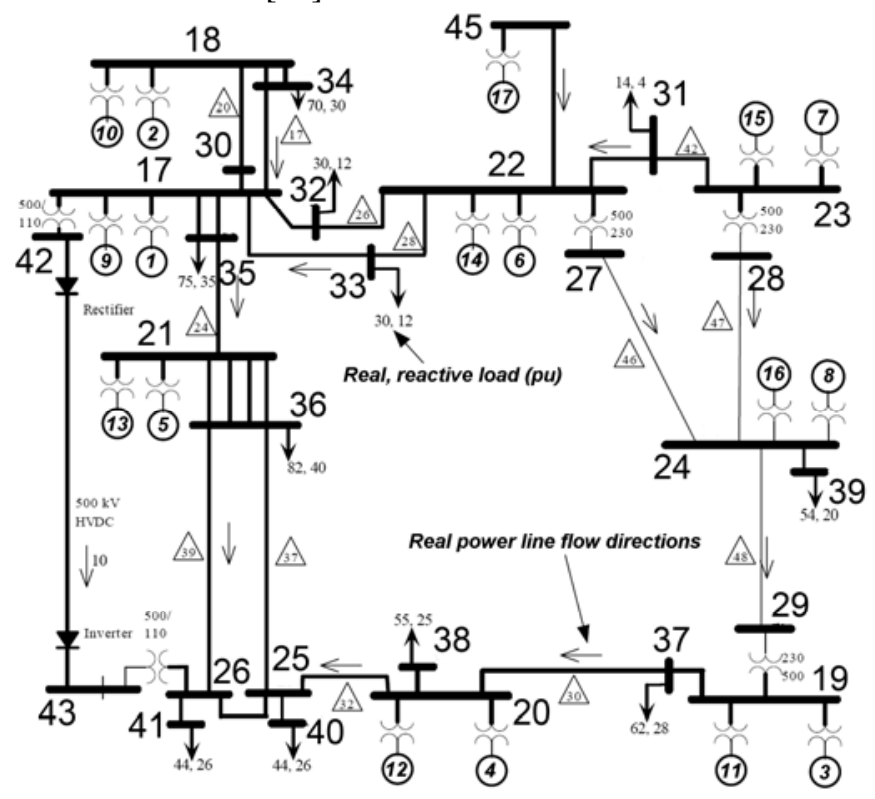

Figure 5-1 - 17-machine model with DC intertie.

To conduct long-term simulations (several minutes), the 17-machine model is linearized into a linear model of order 203 using the MATLAB Power System Toolbox (PST) [3]. The dominant modes of this model are listed in Table 5-1. The mode at $0.422 \mathrm{~Hz}$ and $3.63 \%$ damping is selected for evaluating the performance of recursive Prony algorithm. To generate the ambient data, low-pass filtered Gaussian white noise sequences are used to simulate small real and reactive load changes at all the load buses. Ringdown data is generated through the half-second insertion of a 1400MW brake at bus 35 . The sampling rate of simulation data is set to be $30 \mathrm{samples} / \mathrm{sec}$ to simulate PMU measurement from the WECC Wide Area Measurement Scheme (WAMS). The data set is then decimated to $F_{s}=5$ samples/sec to focus on low frequency mode studies [13].

Table 5-1 - Inter-area modes of 17-machine system

\begin{tabular}{cc|ccc}
\hline \multirow{2}{*}{ Freq (Hz) } & Damp (\%) & \multicolumn{3}{|c}{ Mode Interaction } \\
\hline 0.318 & 10.74 & North half & vs. & Southern half \\
0.422 & 3.63 & North half & vs. & Southern half + bus 45 \\
0.635 & 3.94 & bus 18 & vs. & Rest of the system \\
0.673 & 7.63 & buses 20, 21 & vs. & bus 24 \\
\hline
\end{tabular}

To examine the statistical performance, a Monte-Carlo method is used. The Monte Carlo method uses repeated random sampling to generate a group of data sets for computing estimation results [18]. It is used in the report as follows:

1) Generate $M$ sets of random data to simulate the random load changes. In this report, $M$ is set to be 100 . Each set of data is of 120 seconds in length.

2) Apply each set of random data to the 17 -machine model to simulate random load changes.

3) At $50^{\text {th }}$ second, apply the half-second brake insertion of $1400 \mathrm{MW}$ at bus 35 to generate ringdown data. Apply the proposed ringdown detection method to detect ringdown data.

4) Apply the proposed recursive Prony analysis to identify the power system modes.

The MW power flow on the line from bus 18 to bus 30 was selected for the case studies. A time plot of one output of the 100 Monte Carlo simulations is shown in Figure 5-2 (the DC component has been removed). 


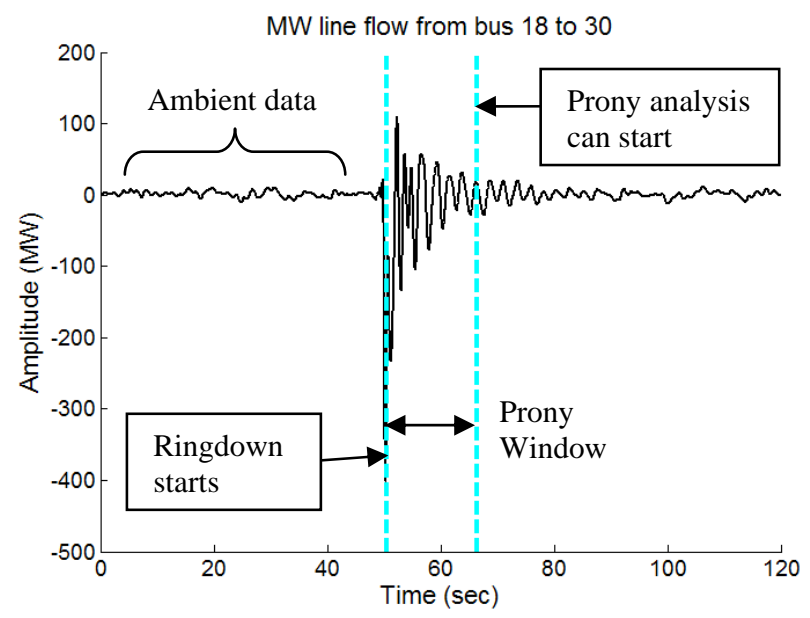

Figure 5-2 - One instant of combined ringdown and ambient data.

The identification parameters are set up as $n=20, N=80$, and $\lambda=1$. Other $\quad$ parameters $\quad$ are $\quad$ set $\quad$ as $\quad \bar{\theta}=0$ and $P[0]=10^{6} \cdot$ Identity Matrix. Note that with selected parameters, the Prony analysis window is set to $N / F_{S}=16$ seconds, so the algorithm is stable only after 16 seconds. Thus, the study results are only plotted out for the time after 16 seconds. Also, due the 16-second time window of Prony analysis, the ringdown data should be accumulated for 16 seconds before Prony analysis can be properly applied. For this simulation study, it means that the Prony analysis should be applicable at about 50.5+16=66.5 seconds, as shown in Figure 5-2.

To evaluate the performance of the proposed ringdown data detection method, the three indices (relative noise level, measurement energy and prediction correction) are calculated for each of the 100 data sets and results are summarized in Figure 5-3 through Figure 5-5. Note that the ringdown detection method is trying to detect when a Prony analysis can be properly applied. As indicated previously, with a 16 second analysis window, this should be around 66.5 seconds.

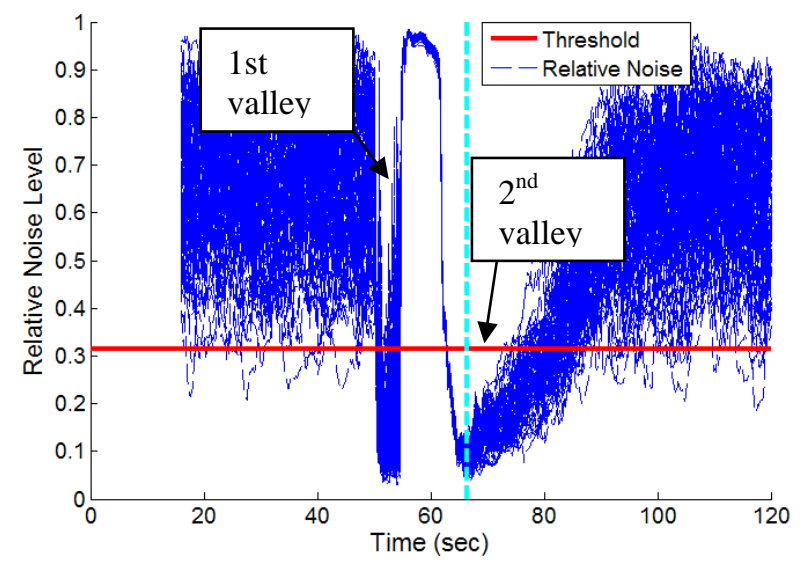

Figure 5-3 - Relative noise levels for 100 data sets of Monte Carlo simulation. 


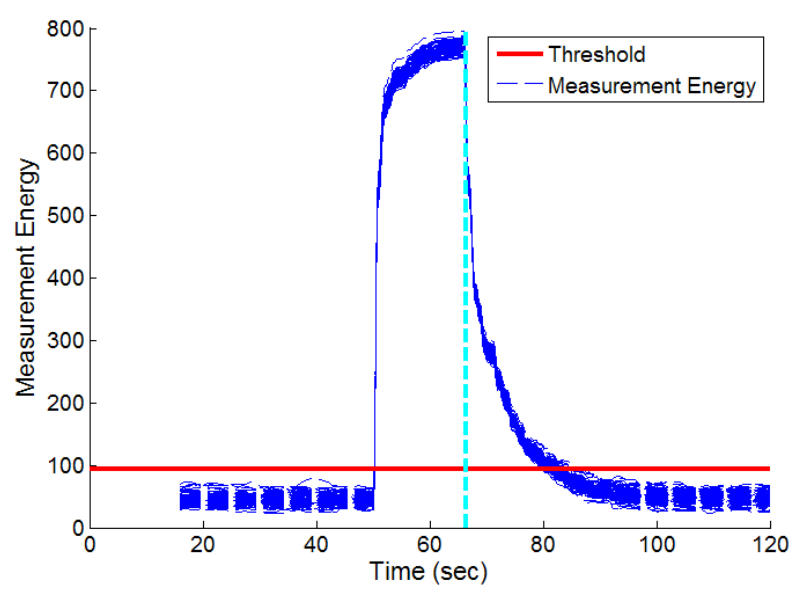

Figure 5-4 - Measurement energy for 100 data sets of Monte Carlo simulation.

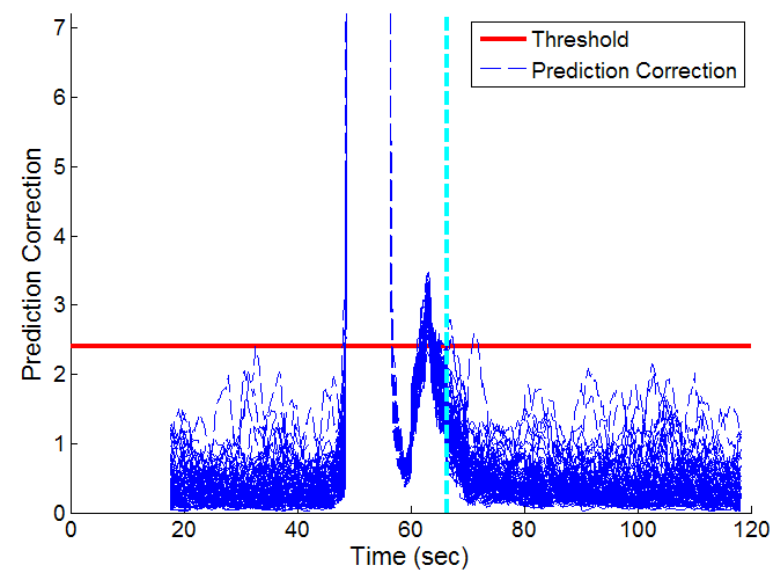

Figure 5-5 - Prediction correction for 100 data sets of Monte -Carlo simulation.

In Figure 5-3, the relative noise levels behave consistently during the ringdown data for 100 sets of data. It has one peak and two valleys. The first valley is due to the over-fit on the initial large transient of the brake insertion. The second valley is where the ringdown signal should be detected. In contrast, the relative noise levels vary significantly for ambient data. Due to the large variance of the relative noise level during the ambient data, detecting ringdown data only based on the relative noise level may result into false detection.

Figure 5-4 shows that the measurement energy for ringdown data is significantly larger than the ambient data portions of the simulations. It can be used to detect ringdown data. Note that detecting ringdown data only based on the measurement energy may result into false detection because any disturbance with large amplitude may lead to large measurement energy.

Figure 5-5 shows that the prediction correction has a peak when the relative noise level in Figure 5-3 has the first valley. It shows that during this period of time, even though the relative noise level is low, mode estimation is not consistent. The adjustment after taking in a new data point is significant. Thus, it is not proper to apply Prony during this period of time. By combining Figure 5-5 with Figure 5-3, the first valley of the Figure 5-5 can be eliminated from ringdown data.

To assist comparison, the three indices are normalized and put together into one figure, shown as Figure 5-6. It can be observed that the three indices show distinguishable behaviors during the ringdown data. Combining the three indices together can result into a reliable detection of ringdown data. 


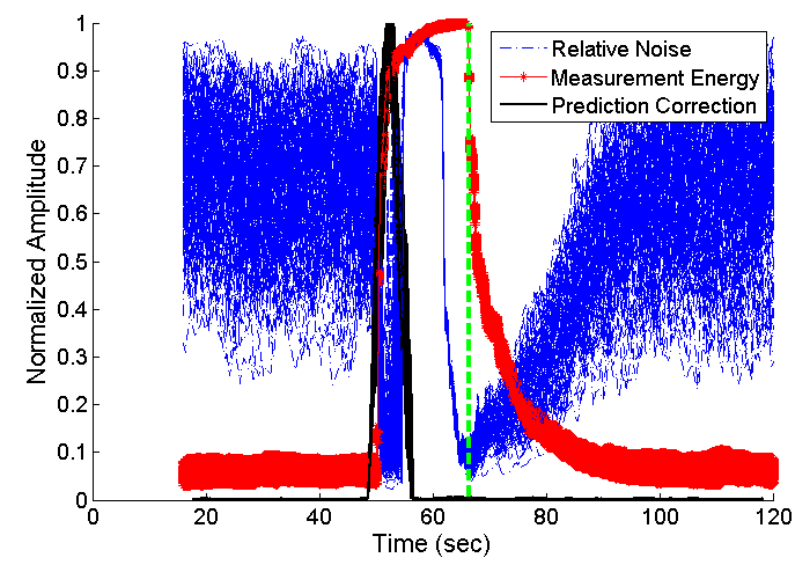

Figure 5-6 - Normalized indices for ringdown data detection.

Applying the proposed method from Section 4.0, the identified ringdown range from 100 data sets is summarized in Figure 5-7. On average, the ringdown detection algorithm indicates the Prony analysis can be started at 66.0 seconds. That indicates that the ringdown data is detected at about 50.0 seconds on average. For 100 sets of simulation data, the standard deviation (std) of ringdown detection time is 0.7 seconds, as indicated by the fainter red lines. It can be observed that the ringdown data is detected with reasonable accuracy. Also, on average, the ringdown data is detected to end at 80.4 seconds. The standard deviation of ringdown ending time is 3.2 seconds, as indicated by the fainter blue lines.

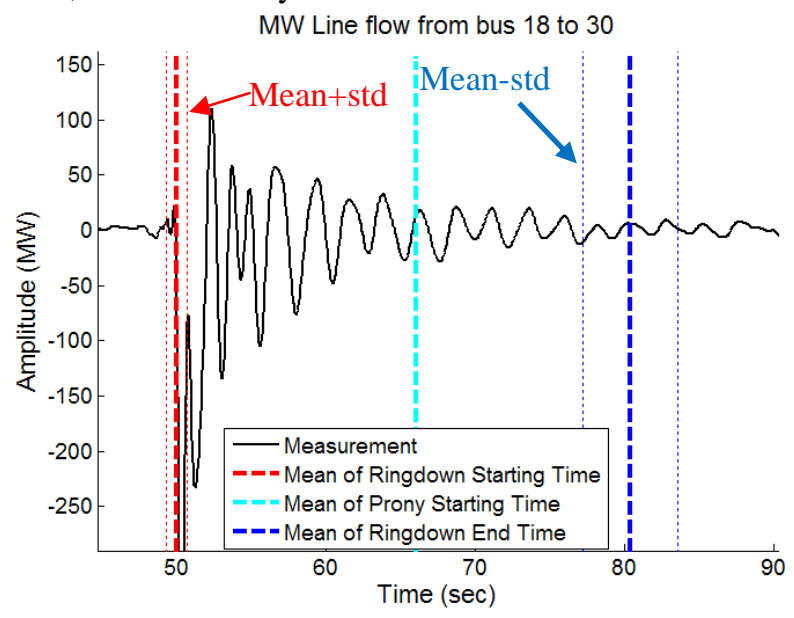

Figure 5-7 - Ringdown detection results.

Applying the proposed Prony analysis over the detected ringdown data using a 16-second window, the mode at $0.42 \mathrm{~Hz}$ can be identified. The results are summarized in Figure 5-8. It can be observed that the mode estimates are clustered around the true mode. It shows that even with 16 seconds of ringdown data, the Prony analysis can provide reasonable mode estimation. In contrast, Figure 5-9 shows the Prony analysis results from ambient data. It can be observed that applying Prony analysis on ambient data with a 16-second window results in large estimation errors. 


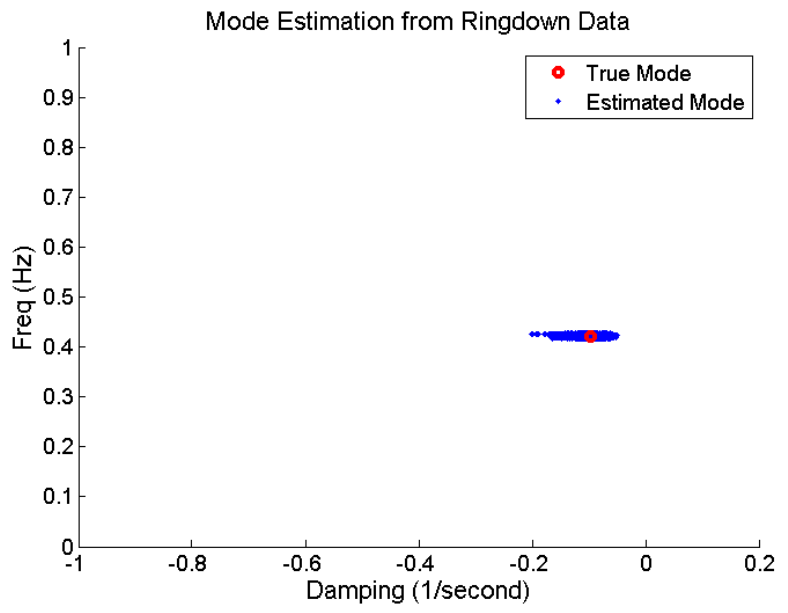

Figure 5-8 - Mode estimation results from detected ringdown data.

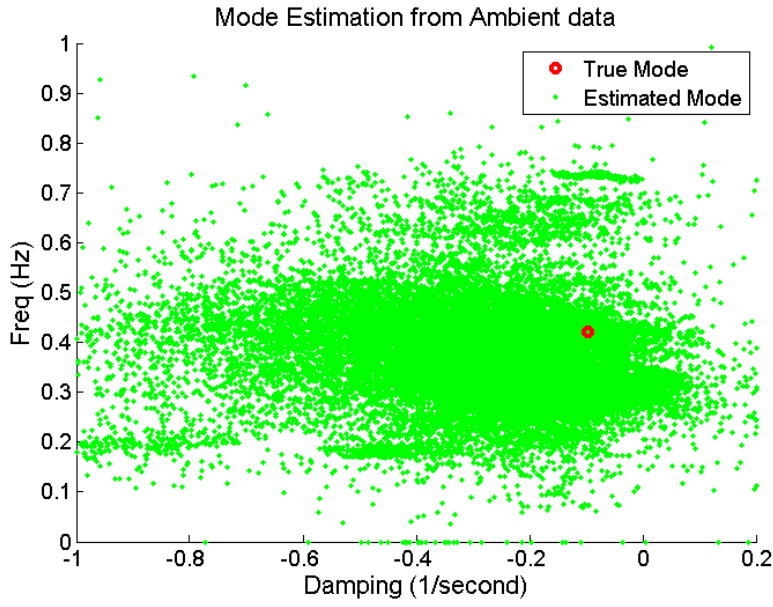

Figure 5-9 - Mode estimation results from ambient data.

In summary, the simulation results show that ringdown data can be effectively detected with the proposed method. Power system modes can be estimated within short time window and within relatively real-time constraints when the recursive Prony analysis is applied to the detected ringdown data. 


\subsection{Field MEASUREMENT Data CASE StUdies}

To further evaluate the performance, the proposed oscillation detection algorithm is applied to the field measured Phasor Measurement Unit (PMU) data. The data are taken from Wide Area Measurement System (WAMS) of WECC system. To protect the data, the DC components are removed and some data are re-scaled.

When detected, oscillations are marked over the time domain plot. In the following figures, the red dashed lines mark the first data point which can be put into Prony study. The magenta dashed lines mark the time when the Prony analysis can be started. Prony analysis can be performed on the data between red and magenta dash line. The distance between the red and magenta line is the Prony analysis window. The Prony window is a constant, which can be selected by users. The Prony window must be large enough so that there are enough data for Prony equation (2) to have solution. The blue dashed line marks the data when the ringdown ends. Prony analysis should be performed recursively over the data between the red and blue dashed lines.

\subsection{Oscillations detected right before WECC Break up on Aug 10th, 1996}

On August 10, 1996, the western North American power grid experienced a system breakup. Later analysis revealed one of the primary causes of the system breakup to be a poorly damped inter-area oscillation around 0.25 Hz [2]. Figure 6-1 shows real power flow on the transmission line from Malin to Round Mountain just before the breakup. Figure 6-1 shows that a number of lines tripped producing intermediate oscillations. The proposed oscillation detection algorithm was applied to this data set to determine when the Prony analysis can be applied to generate accurate mode estimation with relatively short time window.

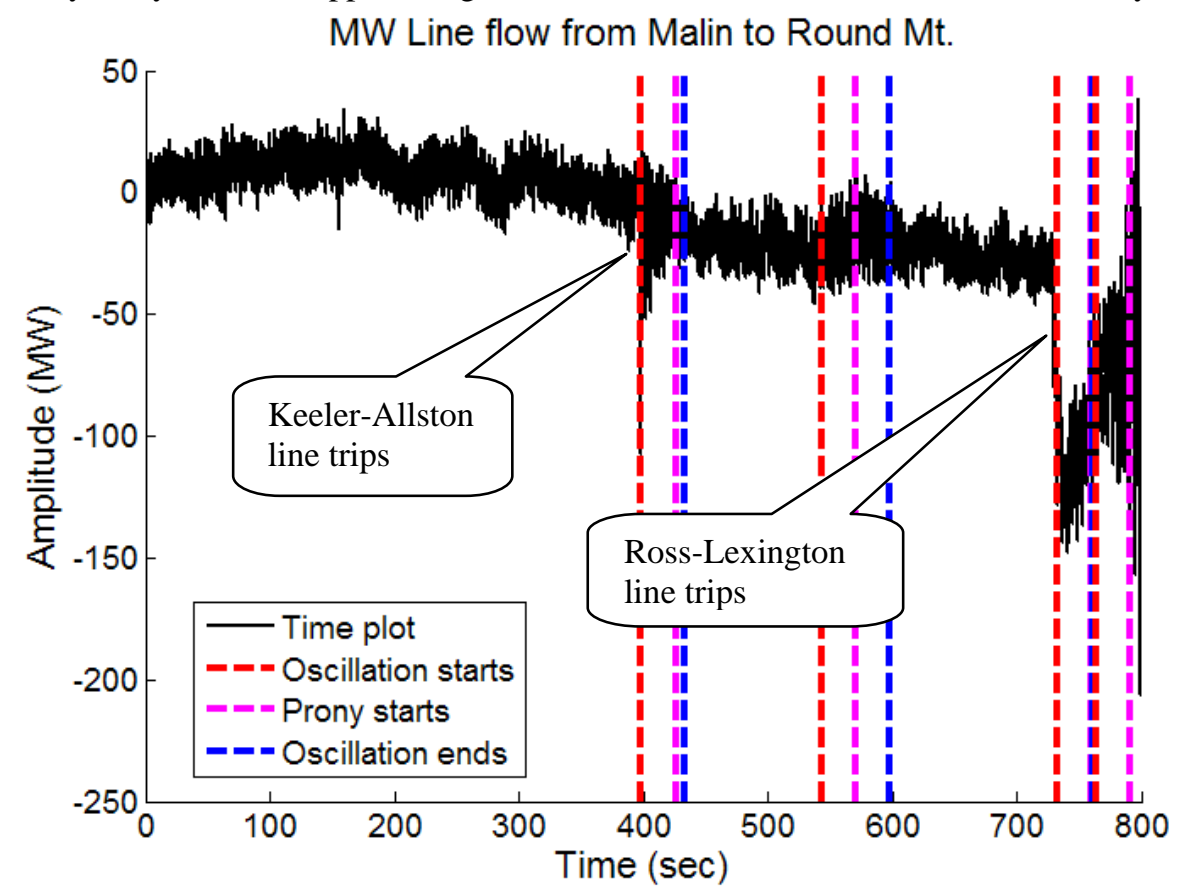

Figure 6-1 - Recorded real power flow from Malin to Round Mountain with detected oscillation. (Reference time: August 10, 1996, 15:35:30 PDT).

As Figure 6-1 demonstrates, according to the algorithm, there are 3 oscillation instances when the Prony analysis can be applied with short time window. The first and last events represent line trips of the Keeler-Allston and Ross-Lexington line, respectively. These events are examined in detail in Figure 6-2 and Figure 6-4. The middle event represents an oscillation on the system caused by device interactions. This oscillation is examined in Figure 6-3. 


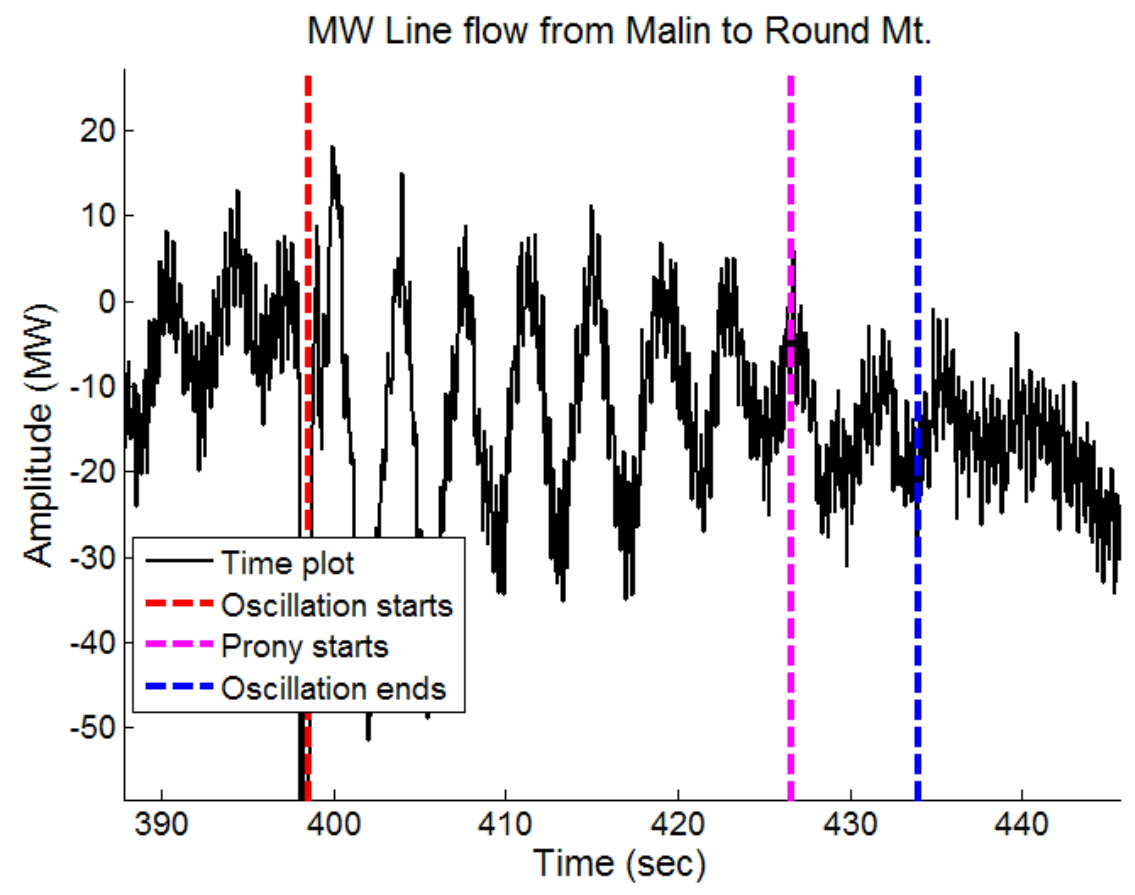

Figure 6-2 - Detail plot of the first oscillation detected after the Keeler-Allston line trips.

Figure 6-2 shows a closer plot of the Keeler-Allston line trip, and the resultant oscillation. As Figure 6-2 indicates, Prony analysis may begin shortly before the 430 second mark of the data. As shown in the data, the algorithm correctly identifies the beginning of the oscillation. The blue dashed-line marks the point when the Prony analysis should be stopped. This line indicates where the ambient noise begins overwhelming the oscillation signal.

MW Line flow from Malin to Round Mt.

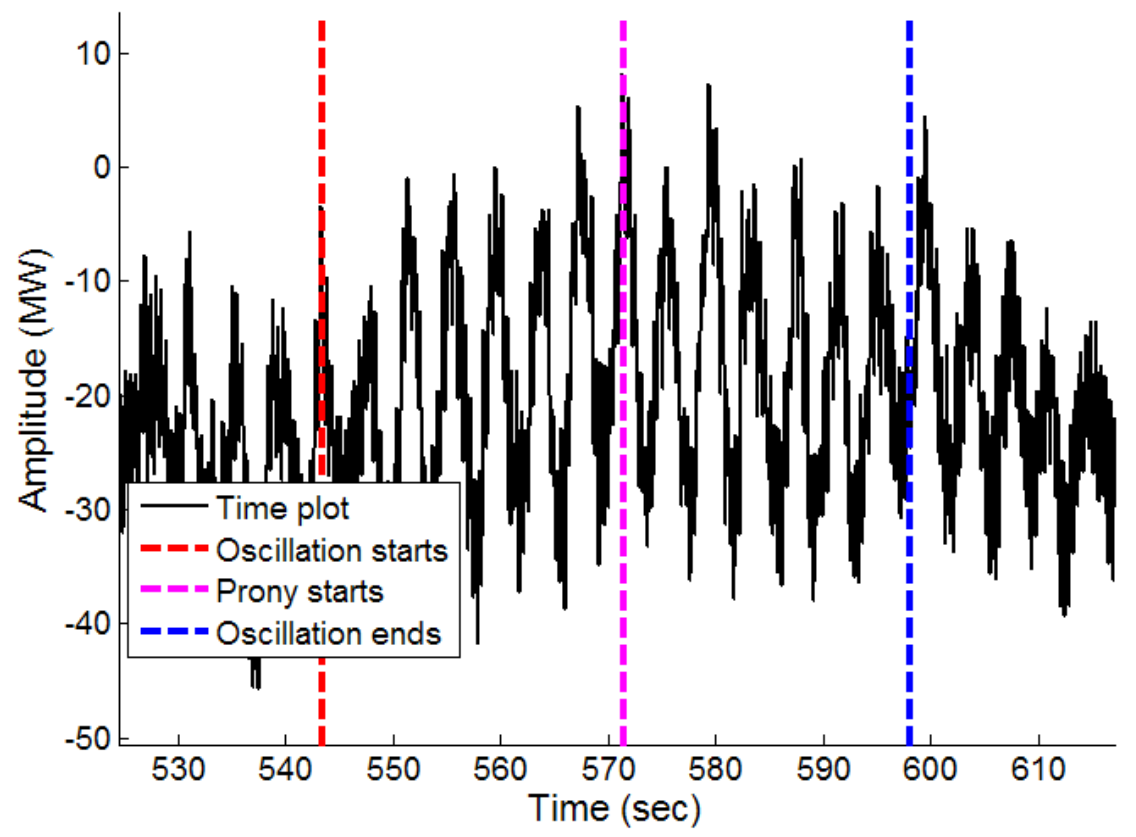

Figure 6-3 - Detail plot of the second oscillation detected.

Figure 6-3 represents a visible oscillation in the original 1996 data that is caused by machine and control responses after the Keeler-Allston line trips. Grid dispatch and event information indicates the previously tripped Keeler-Allston line was unsuccessfully reclosed shortly before this event, so it may be a major influence in the presence of this event. Examining the location of the red line, the oscillation detection algorithm appears to be identifying the start of the oscillation interval correctly. From the raw data of Figure 6-3, the ending oscillation interval (blue line) is not as clear. The algorithm indicates the Prony algorithm should not be applied past roughly the 599 second mark. 


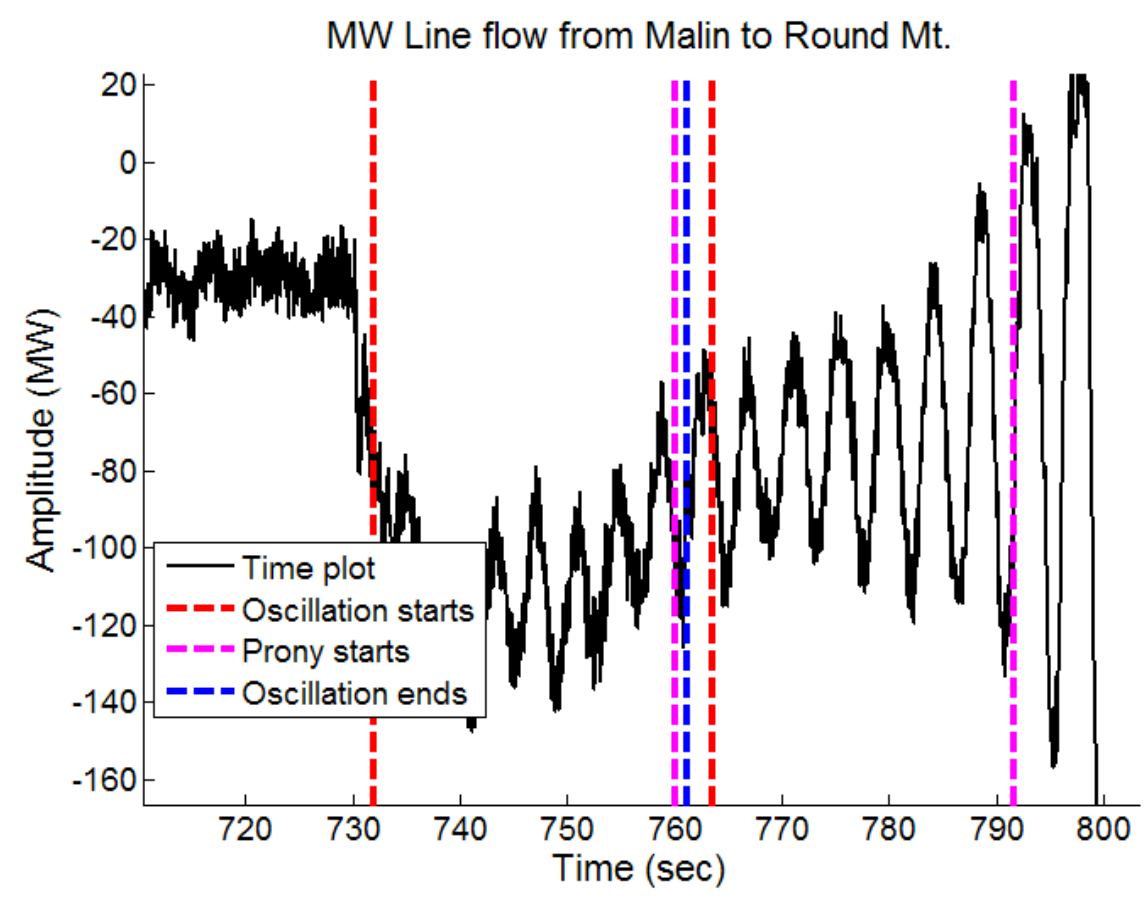

Figure 6-4 - Detail plot of the third and fourth oscillations detected after the Ross-Lexington line trips.

Figure 6-4 shows the result of two other detected oscillations from the August 10, 1996 western interconnection breakup. The first of these two oscillations is associated with the Ross-Lexington line trip. Once again, the oscillation detection algorithm does a good job of detecting the interval to apply Prony analysis. The beginning of the oscillation interval clearly coincides well with the beginning of the oscillation. During this oscillation interval and coinciding with the end of the interval (as indicated by the first blue line), several units of the McNary Dam generation facility were tripping offline. Interestingly, the ringdown ending interval coincides with the tripping of the last unit at McNary. With the complete loss of generation at this facility, the system obviously began to respond in a different way, producing the second oscillation detected.

A second oscillation is detected shortly after the Ross-Lexington trip oscillation. This second oscillation is detected due to a shift in how the system is behaving. Shortly after the first oscillation in Figure 6-4, associated with the Ross-Lexington line trip, the western interconnection began to go unstable and separate itself. This separation is the second oscillation detected in Figure 6-4, and clearly is different from the first oscillation. Despite being a new type of oscillation, the oscillation detection algorithm detects the new interval quickly and indicates a Prony analysis is applicable.

\subsection{Brake Insertion on November 14, 2002}

Ringdown oscillations in the power grid are often associated with lines and generators switching or tripping offline. Another source of oscillations on the power system is the deliberate excitation via a large shunt resistance. Figure 6-5 and Figure 6-6 show the results of inserting a large $1500 \mathrm{MW}$ resistance into the western power system. This $1500 \mathrm{MW}$ resistor, referred to as the Chief Joseph Dynamic Brake, is often switched in momentarily either to aid in improving the stability of the system during machine acceleration major events, or to deliberately excite the power system for dynamic response. 


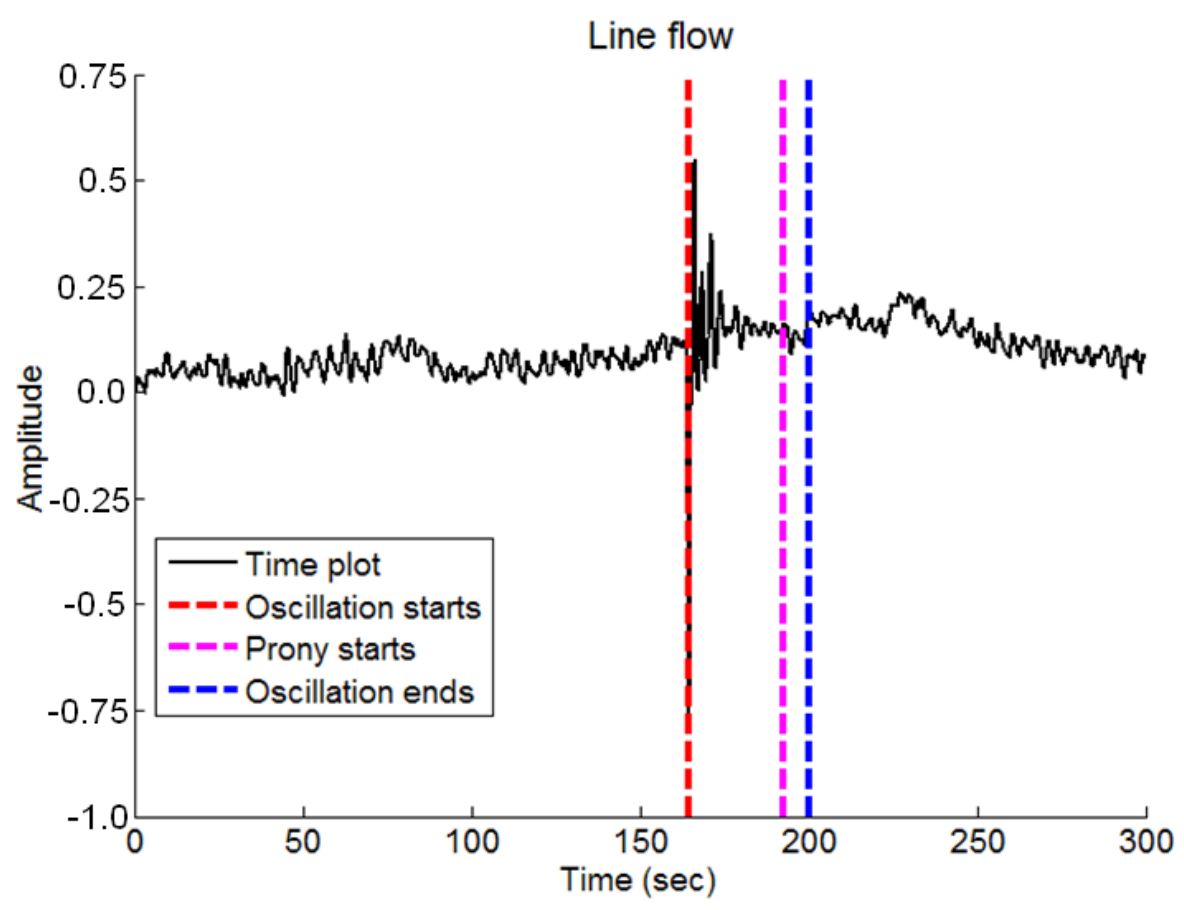

Figure 6-5 - Chief Joseph Dynamic Brake insertion of November 12, 2002.

Figure 6-5 shows an overall view of an insertion of the Chief Joseph Dynamic Brake on November 14, 2002. As the figure demonstrates, the detection algorithm detects the oscillation interval. Unlike the August 10, 1996 case presented previously, the oscillation event is very prominent and easy to spot from the surrounding data. Figure 6-6 shows a closer view of the brake insertion and the resulting detections from the oscillation detection algorithm.

Line flow

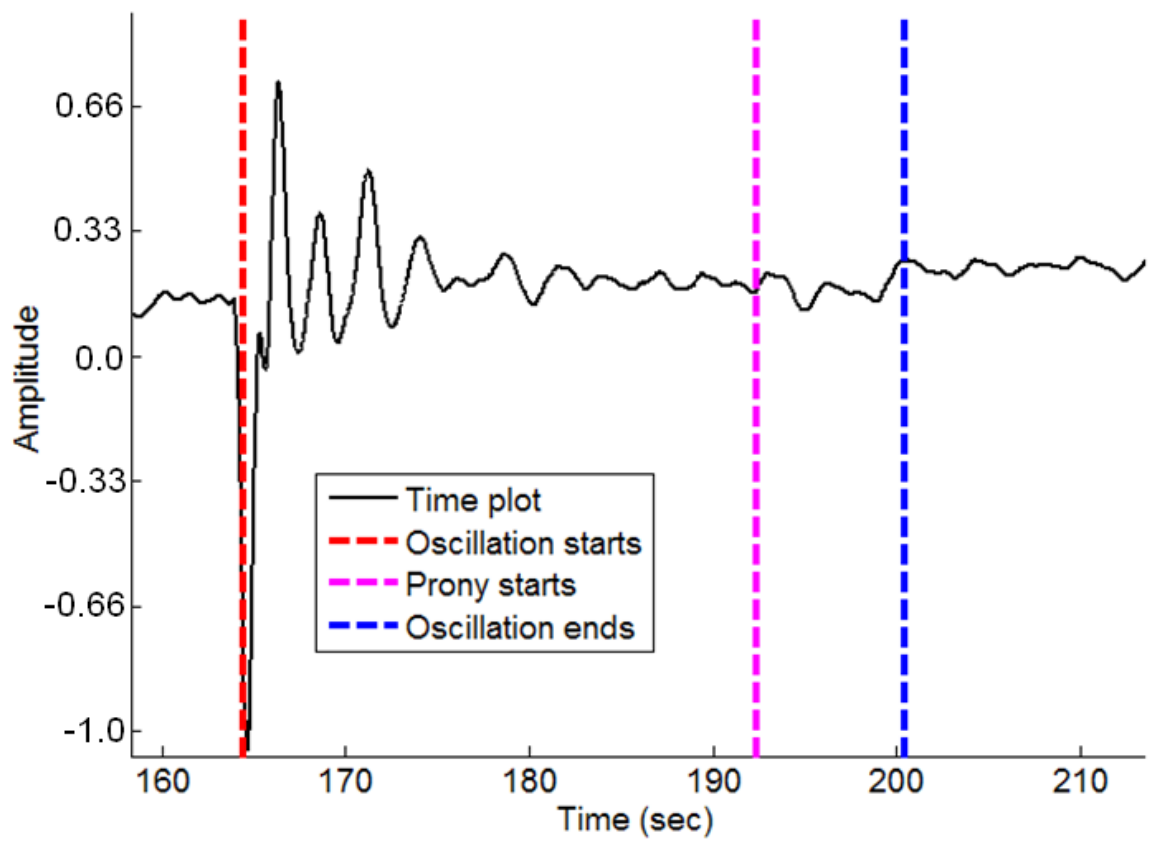

Figure 6-6 - Detail plot of November 14, 2002 Chief Joseph Brake insertion.

Figure 6-6 shows a closer view of the brake insertion, as well as the results of the oscillation detection algorithm's application to those results. As Figure 6-6 demonstrates, the start of the oscillation interval is very nicely detected. An application of Prony analysis from this point would yield a good estimate of the ringdown modal content. Also, the detection algorithm indicates that Prony analysis can continue until approximately the 201 second mark. This indicates that the beginning would be around the 170-second marker of the plot. There are clearly still significant components to the resultant ringdown at this point in the data, so the oscillation algorithm is still successfully detecting a valid Prony analysis range. 


\subsection{Alberta Separation on June 10th, 2002}

As indicated earlier, one of the routine causes of an oscillatory event on the power system are transmission line switching or tripping event. Figure 6-7 shows the measurement data for such an event. On June 10th, 2002, the Alberta, Canada power grid separated from the rest of the WECC grid. As it is shown in Figure 6-7, an oscillation was triggered when the system moved towards a new equilibrium point.

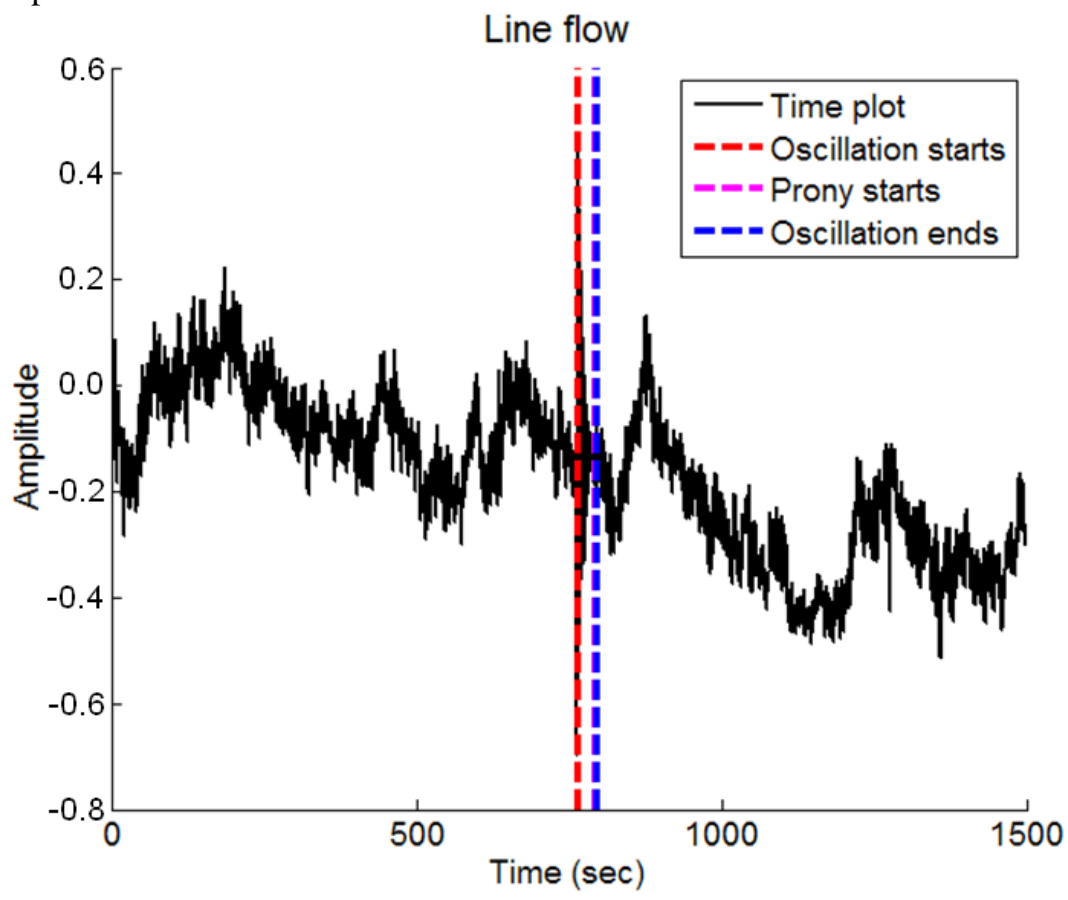

Figure 6-7 - Alberta separation of June 10, 2002.

Figure 6-7 shows the data of the June 10, 2002 Alberta separation event. This event is not very prominent visually in the data interval presented. Without prior knowledge of the event or the oscillation detection algorithm, the event would easily be overlooked. Figure 6-8 shows a closer view of the event and the oscillation detection algorithm results.

Line flow

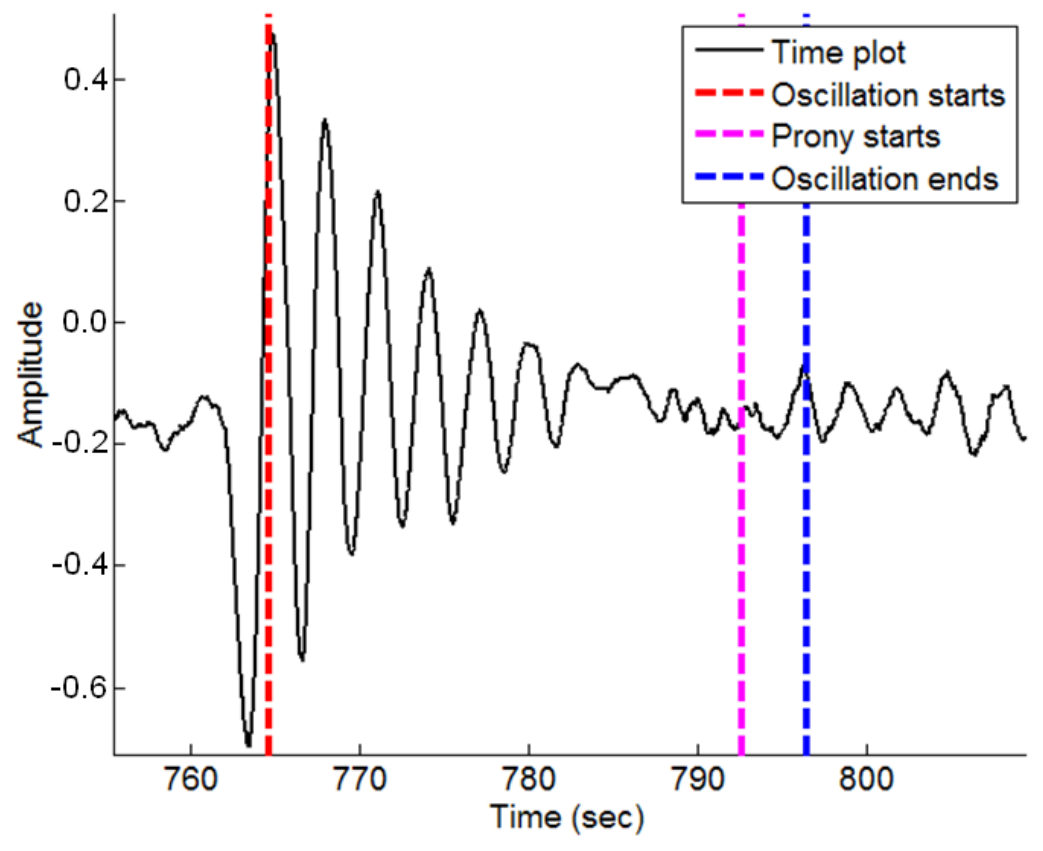

Figure 6-8 - Detail plot of Alberta separation of June 10, 2002.

Upon closer inspection, there is clearly an oscillatory event associated with the Alberta separation. As Figure 6-8 shows, the oscillation detection algorithm selects a reasonable interval for valid Prony analysis. The beginning of the selected interval is a 
valid starting point for Prony analysis and the oscillation ending interval is reasonable given the data. It is important to point out that this analysis has a much shorter interval than the previous brake insertion example. As Figure 6-7 demonstrated, the Alberta separation ringdown is much smaller in amplitude, so it does not contain as much energy in comparison with ambient noise.

\subsection{Palo Verde Generation Trip on Nov 18, 2000}

Generator tripping events are also a prominent cause of oscillatory events on the power system. On November 18, 2000, a loss of generation occurred at the Palo Verde generation facility. Figure 6-9 shows the measurement data for this loss of generation.

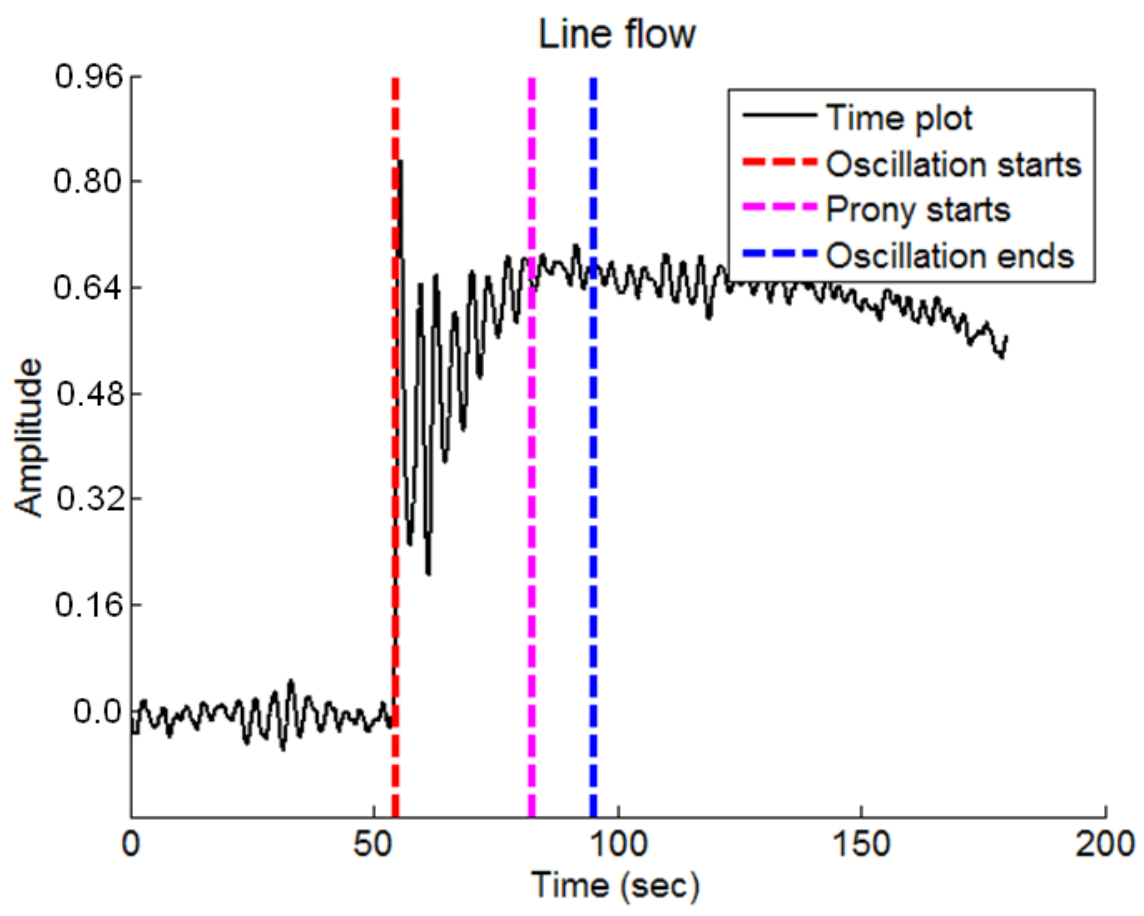

Figure 6-9 - Palo Verde generation trip of November 18, 2000.

As with the previous figures, the results of the oscillation detection algorithm are overlaid in Figure 6-9. With a large response to the loss of the generation, the oscillation detection algorithm had little trouble locating the proper interval to begin Prony analysis. The oscillation detection algorithm indicated Prony analysis would remain valid until the interval ending around 95 seconds. If the oscillation start time is offset by this amount, significant components of the generation trip's oscillation will still be present in the analysis interval.

\subsection{PDCI Block on November 2, 2004}

Different oscillations can be induced by normal transmission lines tripping. Figure 6-10 shows the high voltage, long DC switching event that occurred on November 2, 2004. During this event, there was a planned adjustment on the Pacific DC Intertie that resulted in a readjustment of the amount of power that the parallel AC lines were carrying. 


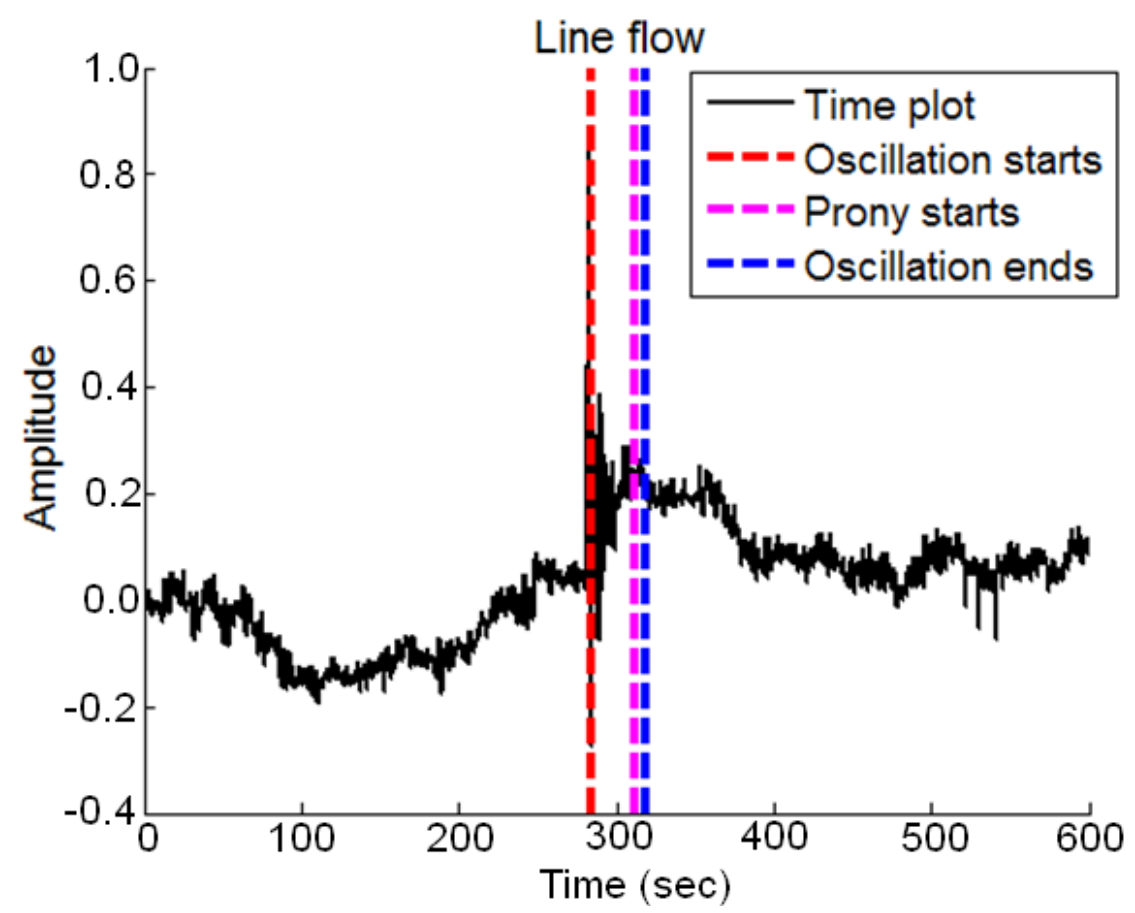

Figure 6-10 - Pacific DC Intertie block even of November 2, 2004.

Figure 6-10 shows the oscillation event caused by the PDCI block. A closer examination of the oscillation is shown in Figure 6-11. As with some of the previous examples, the event is prominent when compared to the surrounding data. However without explicit knowledge of when the event occurred and without the use of the oscillation detection algorithm, this event could easily be missed during normal grid operations.

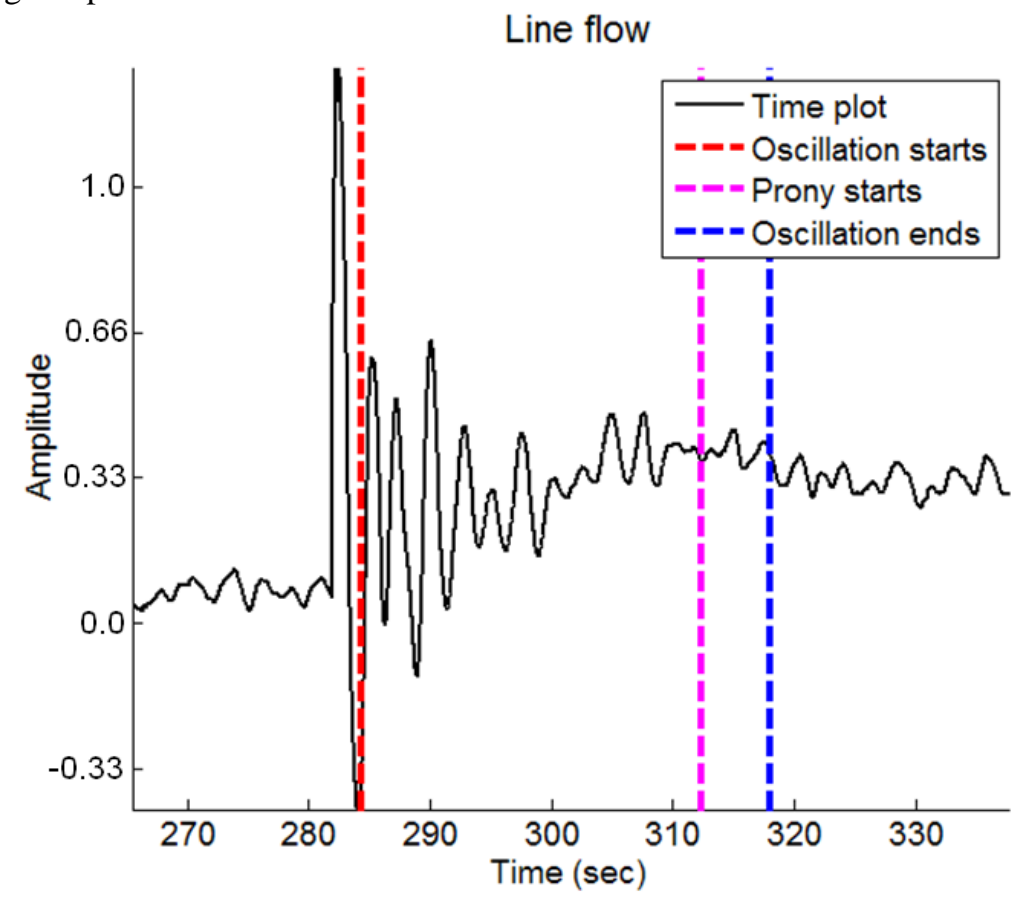

Figure 6-11 - Detail plot of PDCI block event of November 2, 2004.

Figure 6-11 shows the results of the oscillation detection algorithm. The algorithm is clearly detecting the ringdown associated with the PDCI event. From Figure 6-11, it appears the oscillation analysis begins a cycle too late. However, the significantly larger swings of the first cycle of the oscillation are not at the same frequency as the rest of the ringdown, so the detection algorithm does not consider this oscillation to be valid for the ringdown analysis. As with other detections, once the ringdown event begins to have amplitudes similar to the rest of the power system data, the algorithm indicates the Prony analysis interval is complete. 
6.6. Brake Insertion following Alberta Separation on July 24, 2006

In an earlier example, an event caused by the separation of Alberta, Canada from the rest of the WECC was examined. Figure 6-12 represents another Alberta separation from July 24, 2006. Unlike the previous example, the separation is barely visible in Figure 6-12. The large, initial oscillation in Figure 6-12 is predominantly a dynamic brake insertion following the faulting of another line in the WECC system. This Alberta separation further differs from the earlier example in that a secondary event later in the data is present.

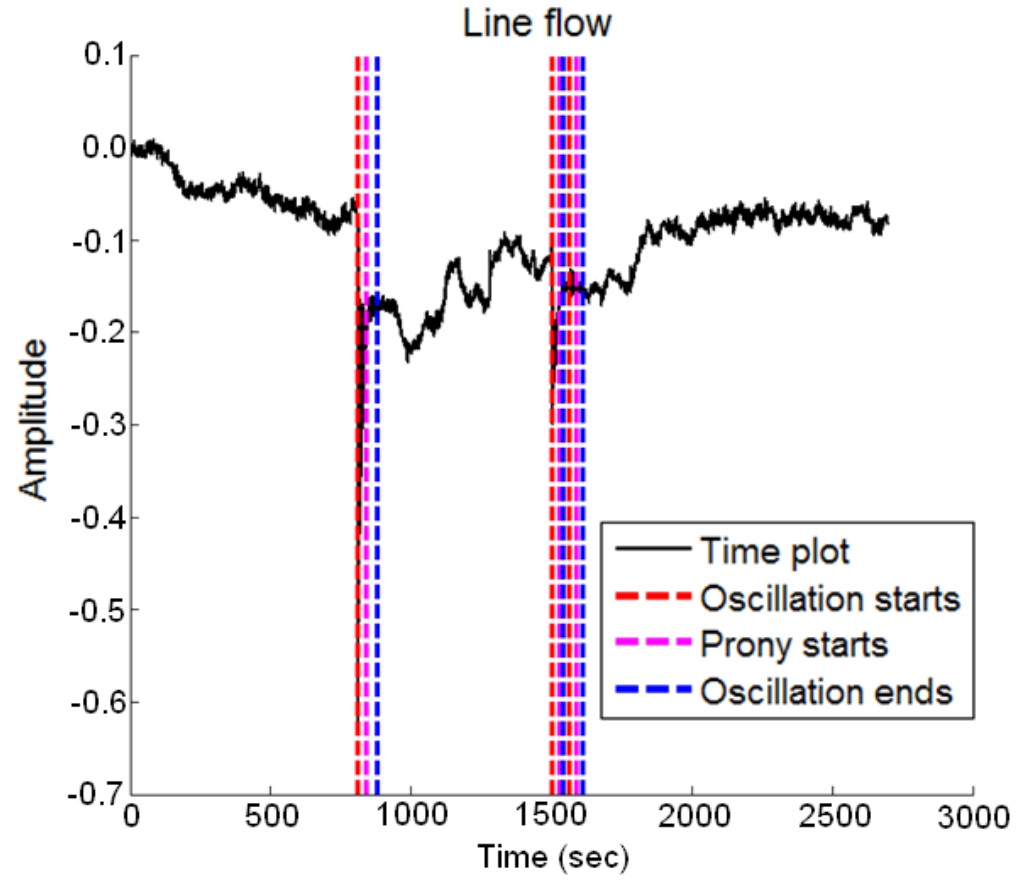

Figure 6-12 - Alberta separation of July 24, 2006.

Figure 6-12 shows the overall oscillation detection algorithm results for the Alberta separation event. As the figure indicates, there are actually three oscillations detected over the data interval. The first event is shown in greater detail in Figure 6-13. The second and third events are shown in Figure 6-14.

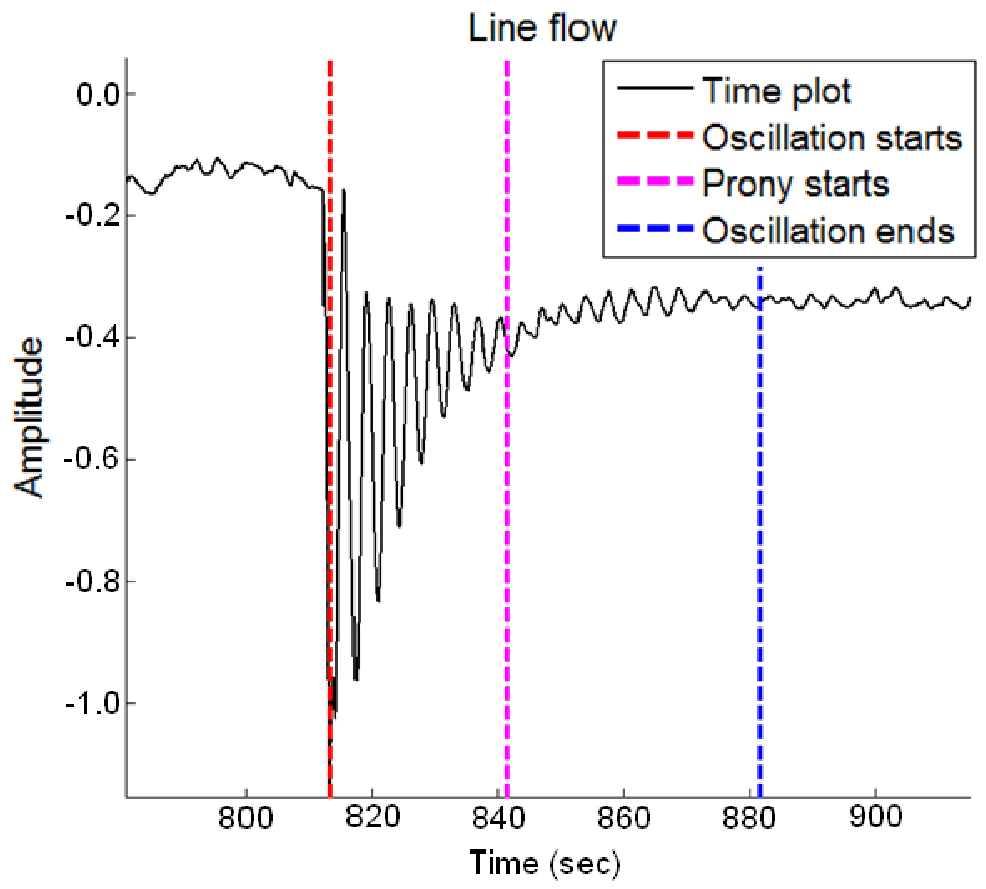

Figure 6-13 - Detail plot of line fault and brake insertion shortly after Alberta separation of July 24, 2006. 
The first event of the Alberta separation data set represents a line fault and the insertion of the Chief Joseph dynamic brake. The actual Alberta separation occurs around the 810 second mark and is not prominent on the data, so it was not detected by the oscillation detection algorithm. Figure 6-13 shows the oscillation that resulted when the Chief Joseph braking resistor was briefly inserted into the system. The oscillation detection algorithm successfully detects the start of a suitable Prony analysis interval. The analysis interval appears to extend well beyond the ringdown data. However, closer examination of Figure 6-13 reveals a secondary oscillation event over the 850 and 880 second time interval. Given the larger amplitude of the initial oscillation, the secondary oscillation could easily have been present, but not apparent in the signal at that time.

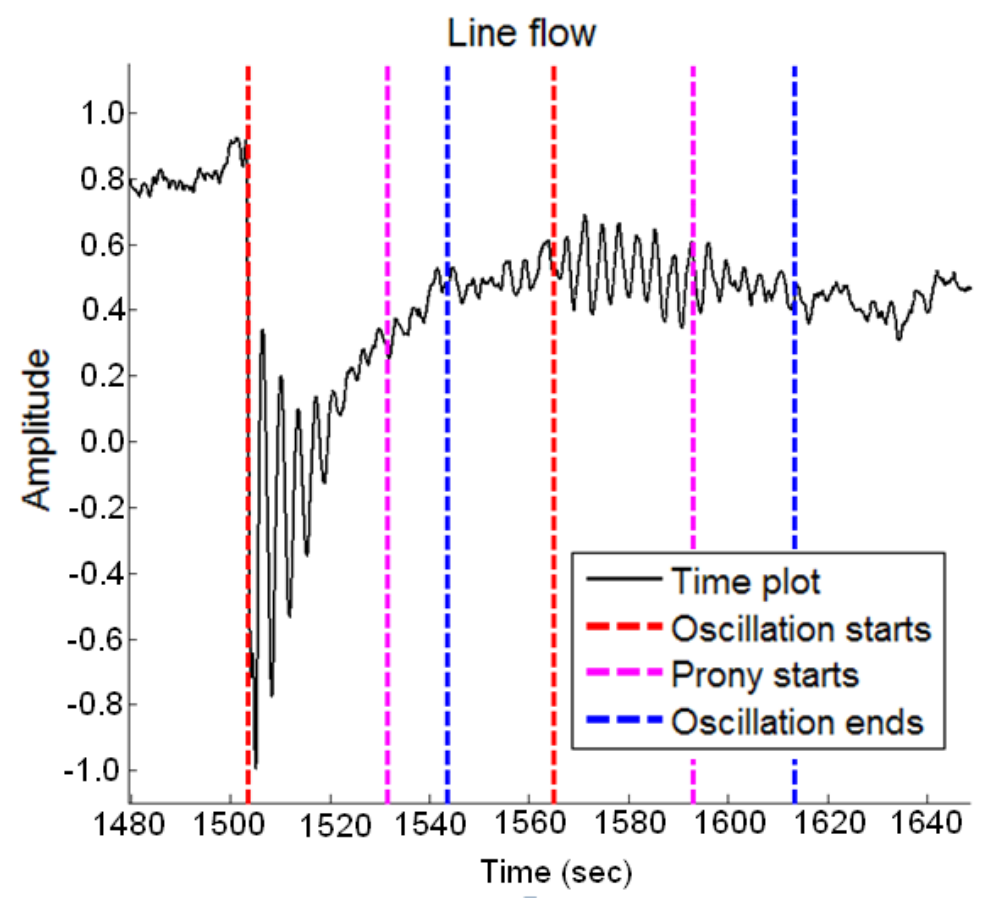

Figure 6-14 - Detail plot of Colstrip generation loss and secondary event after Alberta separation of July 24, 2006.

Along with the primary, line-trip event association with the Alberta separation, a secondary series of events were also observed. Figure 6-14 shows the oscillatory events caused by a generation trip and later responses to that tripping event. The oscillation successfully detected at the 1505 second mark was caused by a trip of a generator at the Colstrip generation facility. Roughly a minute later, other, smaller events occurred on the system. While the cause of these oscillation is uncertain at this time, they may be related to the switching out of a shunt capacitor bank. Despite not being as prominent as the initial generator trip event, Figure 6-14 shows these events were clearly oscillatory in nature and successfully detected by the algorithm.

\subsection{California Machine Control event on January 4, 2010}

As indicated earlier in the examples, line trip events and system separations are not the sole cause of oscillatory behavior on the system. Generators can also produce oscillatory responses on the system. Figure 6-15 shows an oscillation caused by a California generator on January 4, 2010. As Figure 6-15 and the detail plot in Figure 6-16 show, the event is very prominent, but is not started by an abrupt shift like most of the other oscillation examples presented. 


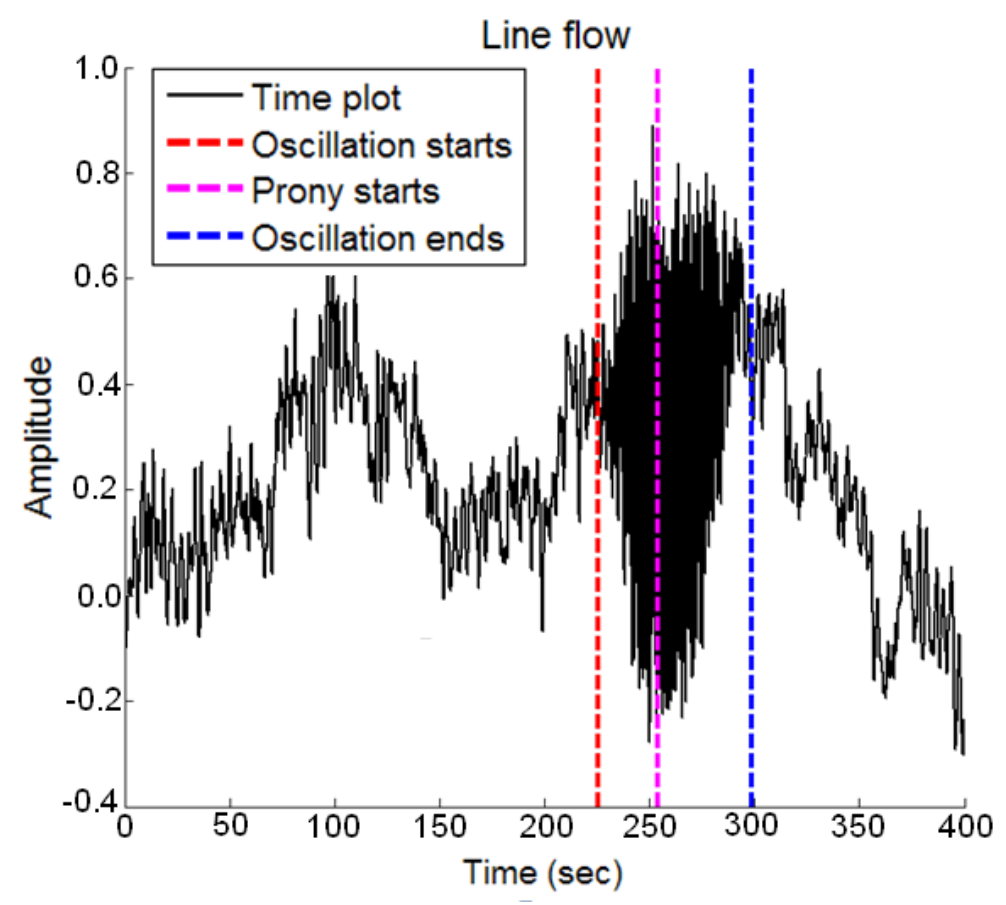

Figure 6-15 - California machine control event of January 4, 2010.

As Figure 6-15 shows, the generation oscillation event is visually prominent on the measured data. Unlike the line tripping and system separation events, there is no discrete cause of the oscillation. Rather, the oscillation amplitude slowly increases in response to the event. Despite the lack of a discrete oscillation start, the oscillation detection algorithm still locates suitable starting and ending times for ringdown analysis of the event. A detail plot of the oscillation detection times is shown in Figure 6-16.

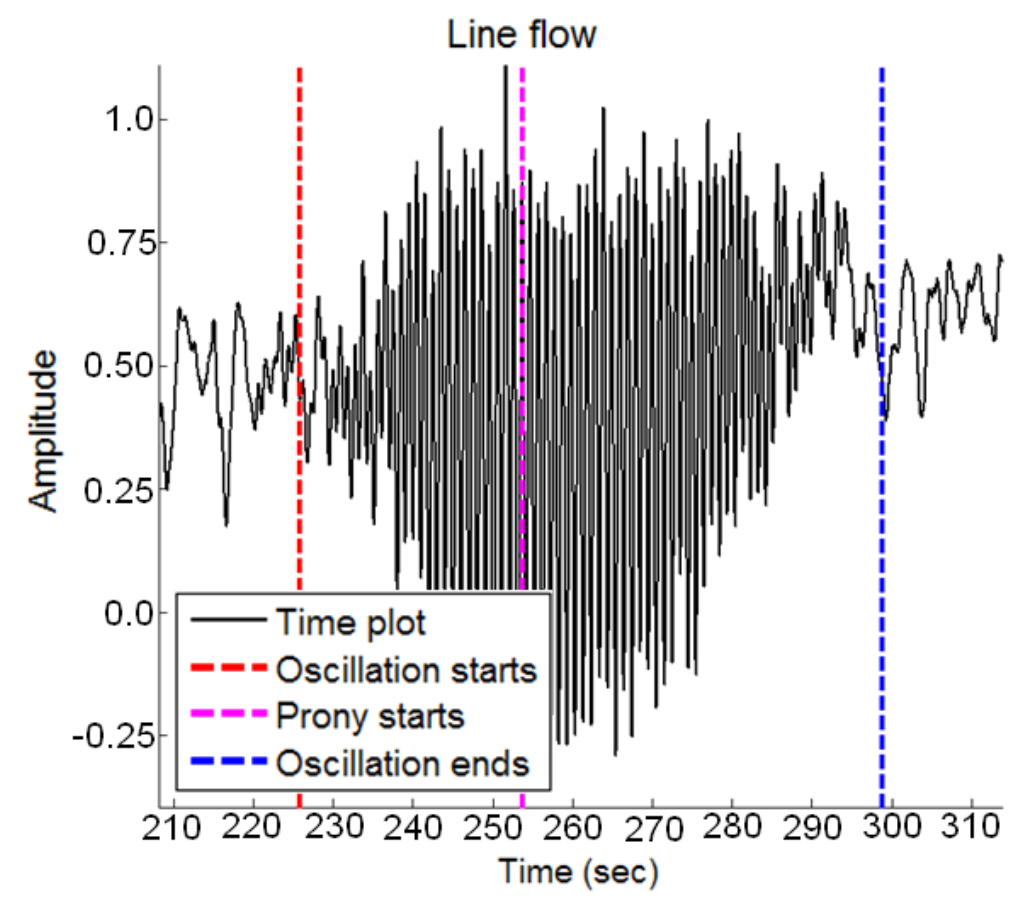

Figure 6-16 - Detail plot of California machine control event of January 4, 2010.

The oscillation is very prevalent in the detail plot of Figure 6-16. Despite the higher frequency of the oscillation produced by this event, the detection algorithm produces a valid Prony analysis interval. Examination of the plot indicates the oscillation may have started a few seconds before the red, oscillation start line the algorithm determined. However at this point in the data, the oscillation is still at approximately the same amplitude as the ambient power system noise. This smaller amplitude and less consistent nature of the oscillation required a couple seconds more data before the detection algorithm indicates a valid interval. 
Despite this possibly delayed starting interval, the ringdown stop interval appears very well selected with the end of the ringdown associated with the event.

Figure 6-15 and Figure 6-16 represent the application of the ringdown detection algorithm over a short data interval. For a practical dispatch center application, the ringdown oscillation detector would be running over the full 24hours of the day. To evaluate this performance, the ringdown oscillation detection algorithm was run on the full January 4, 2010 data set. According to dispatch records, the California machine control event was the only disturbance recorded over the 24-hour period. The ringdown detection algorithm only detects one event over the 24-hour interval as well. Furthermore, some measurement errors were present in the data. The ringdown oscillation detection algorithm successfully ignores these non-typical data points and does not register a false alarm. Figure 6-17 shows the detail of the detection.

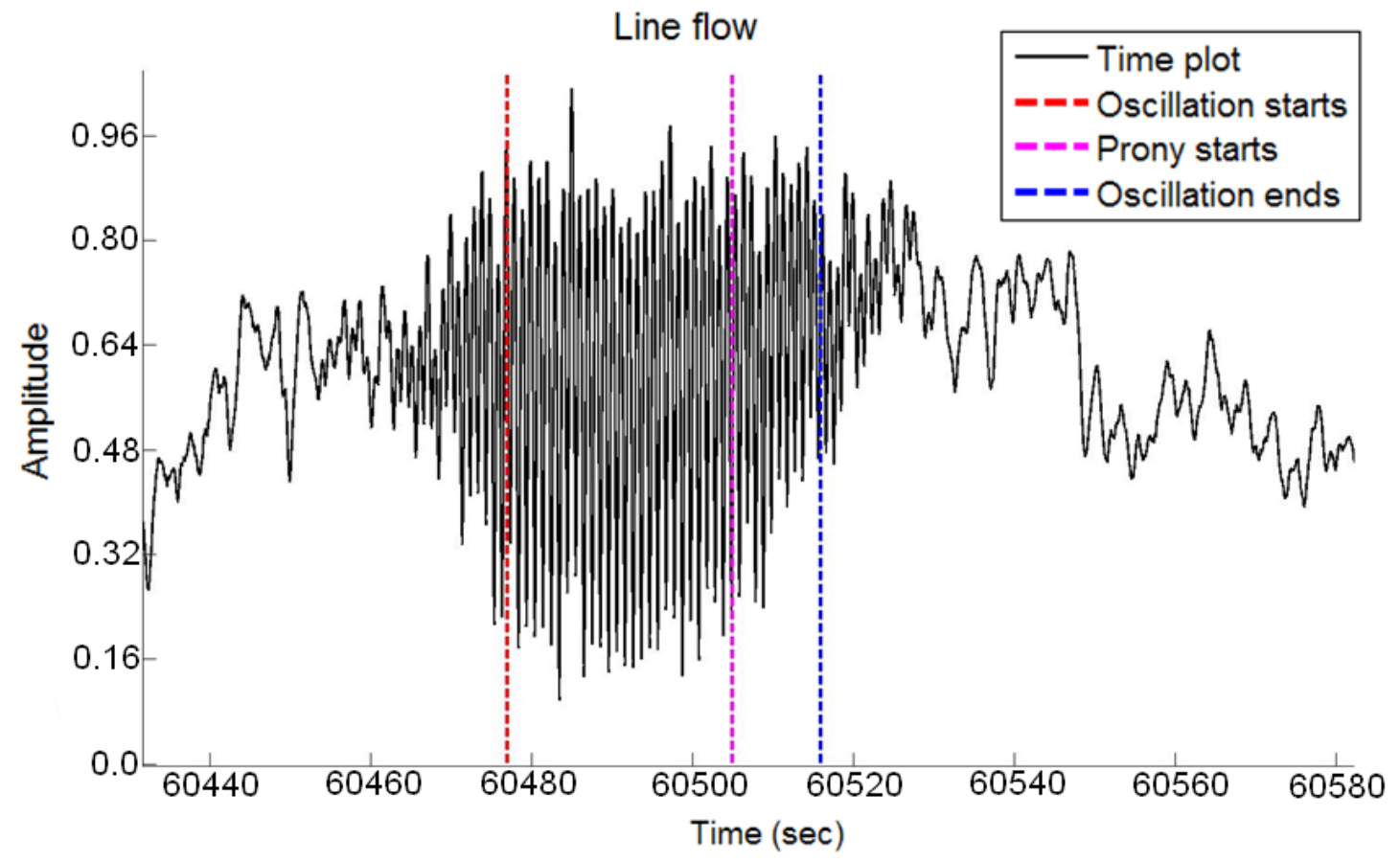

Figure 6-17 - Detail plot of California machine control event of January 4, 2010 - 24 hour data

As Figure 6-17 shows, the ringdown oscillation detection algorithm still detects the California machine control event, despite having been running for approximately 18 hours already. As the figure shows, the detected analysis interval is slightly different than that of Figure 6-16. This is primarily a result of the longer data set. After running for 18 hours, the detection algorithm has a different estimate of the relative noise of the system. As such, the increasing amplitude of the oscillation takes more time to resolve than the shorter data set case. This also explains the shorter Prony interval compared to Figure 6-16, as shown by the blue dashed-line appearing earlier. Despite these differences, the algorithm still detected a sufficient interval to apply the Prony analysis method. 


\subsection{CONCLUSIONS AND FUTURE WORK}

This report developed and evaluated a recursive algorithm of Prony analysis for on-line monitoring inter-area modes based on simulation and field measurement PMU data.

Modal analysis provides vital information about the power system stability. On-line mode identification algorithms based on PMU measurements provide a way for monitoring power system modes in real time. Oscillation alarms can be issued when the power system is lightly damped. A good oscillation alarm tool (a.k.a. ModeMeter) can provide time for operators to take remedial reaction and reduce the probability of a system breakup as a result of such a light damping condition.

The applicability of the mode identification algorithms relies heavily on the proper use of algorithms. Identification algorithms can provide dependable mode information only when they are applied properly on the right signal types. Improper application of algorithms may result in false alarms and/or missing alarms.

One of most import categories of data is the oscillation ringdown data, which results from major disturbances. It is important to detect the oscillation ringdown to properly apply Prony’s method to promptly identify modes within a short time window.

In this report, a method for detecting ringdown data is proposed and evaluated. Through Monte Carlo simulation, it was shown that Prony analysis can be applied automatically and properly on the detected ringdown data to estimate the power system modes. The evaluation based on the field measurement shows that the proposed method is applicable to the measurement data. By effectively identifying ringdown data, the modes can be identified accurately within a short time window. Thus, the detection reduces the rate of false and missing alarm. 


\subsection{BIBLIOGRAPHY}

[1] Pal, B. and B. Chaudhuri, Robust Control in Power Systems, Springer US, 2005

[2] Kosterev, D. N., C. W. Taylor, and W. A. Mittelstadt, "Model Validation for the August 10, 1996 WSCC System Outage," IEEE Transactions on Power Systems, vol. 14, no. 3, pp. 967-979, August 1999.

[3] Chow, J., Power System Toolbox, Dynamic Tutorial and Functions, 1997.

[4] Hauer, J.F., C. J. Demeure, and L.L. Scharf, “Initial Results in Prony Analysis of Power System Response Signals,” IEEE Transactions on Power Systems, vol. 5, no. 1, pp. 80-89, Feb. 1990.

[5] Liu, G., J. Quintero, and V. Venkatasubramanian, “Oscillation Monitoring System based on wide area synchrophasors in power systems,” Proc. IREP symposium 2007. Bulk Power System Dynamics and Control - VII, August 19-24, 2007, Charleston, South Carolina, USA.

[6] Liu G., and V. Venkatasubramanian, "Oscillation monitoring from ambient PMU measurements by Frequency Domain Decomposition," Proc. IEEE International Symposium on Circuits and Systems, Seattle, WA, May 2008, pp. 2821-2824.

[7] Pierre, J.W., D.J. Trudnowski, and M. K. Donnelly , "Initial results in electromechanical mode identification from ambient data," IEEE Transactions on Power Systems, vol.12, no.3, pp. 1245-1251, Aug. 1997.

[8] Kamwa, I., G. Trudel, and L. Gerin-Lajoie, "Low-order Black-box Models for Control System Design in Large Power Systems," IEEE Transactions on Power Systems, vol. 11, no. 1, pp. 303-311. Feb. 1996

[9] Messina, A. and Vijay Vittal, "Nonlinear, non-stationary analysis of interarea oscillations via Hilbert spectral analysis," IEEE Transactions on Power Systems, vol. 21, no 3, pp. 1234-1241, Aug, 2006.

[10] Trudnowski, D., J. Pierre, N. Zhou, J. Hauer, and M. Parashar, "Performance of Three Mode-Meter Block-Processing Algorithms for Automated Dynamic Stability Assessment," IEEE Transactions on Power Systems, vol. 23, no. 2, pp. 680-690, May 2008.

[11] Sanchez-Gasca, J. J., and J. H. Chow, "Performance Comparison of Three Identification Methods for the Analysis of Electromechanical Oscillations", IEEE Transactions on Power Systems, vol. 14, no. 3, pp. 995-1001. Aug. 1999.

[12] Zhou, N., J. Pierre, and J. Hauer, "Initial Results in Power System Identification from Injected Probing Signals Using a Subspace Method,” IEEE Transaction on Power Systems, vol. 21, no. 3, pp. 1296-1302, Aug 2006.

[13] Zhou, N., J. Pierre, D. Trudnowski, and R. Guttromson, "Robust RLS Methods for On-line Estimation of Power System Electromechanical Modes,” IEEE Transactions on Power Systems, vol. 22, no. 3, Aug. 2007, pp. 1240-1249.

[14] Zhou, N., D. Trudnowski, J. Pierre, and W. Mittelstadt, "Electromechanical Mode On-Line Estimation using Regularized Robust RLS Methods", IEEE Transactions on Power Systems, vol. 24, no. 4, pp. 1670-1680, November 2008

[15] Zhou, N., D. Trudnowski, and J. Pierre, "Mode Initialization for On-Line Estimation of Power System Electromechanical Modes," Proceedings of the 2009 Power Systems Conference and Exposition (PSCE), Seattle, WA, March 15-19, 2009.

[16] Ljung, L., System Identification: Theory for the User, 2nd edition, Prentice Hall, Upper Saddle River, New Jersey, 1999.

[17] Trudnowski, D., M. Donnelly, and E. Lightner, "Power-System Frequency and Stability Control using Decentralized Intelligent Loads," Proceedings of the 2005/2006 IEEE PES T\&D Conference and Exposition, Dallas, TX, pp. 1453-1459, May 2006.

[18] Wikipedia contributors. (November 2009) Monte Carlo method. Accessed November 23, $2009 . \quad$ [Online]. Available: http://en.wikipedia.org/wiki/Monte_Carlo_method 